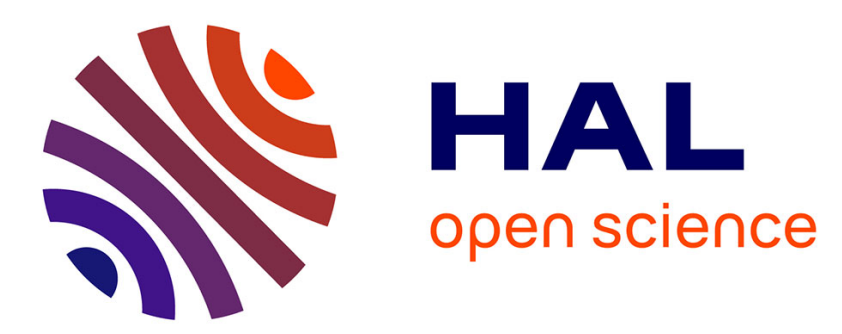

\title{
Cosmological constraints from CODEX galaxy clusters spectroscopically confirmed by SDSS-IV/SPIDERS DR16
}

J Ider chitham, J Comparat, A Finoguenov, N Clerc, C Kirkpatrick, S Damsted, A Kukkola, R Capasso, K Nandra, A Merloni, et al.

\section{To cite this version:}

J Ider chitham, J Comparat, A Finoguenov, N Clerc, C Kirkpatrick, et al.. Cosmological constraints from CODEX galaxy clusters spectroscopically confirmed by SDSS-IV/SPIDERS DR16. Monthly Notices of the Royal Astronomical Society, 2020, 499 (4), pp.4768-4784. 10.1093/mnras/staa3044 . hal-02991967

\section{HAL Id: hal-02991967 https://hal.science/hal-02991967}

Submitted on 27 Nov 2020

HAL is a multi-disciplinary open access archive for the deposit and dissemination of scientific research documents, whether they are published or not. The documents may come from teaching and research institutions in France or abroad, or from public or private research centers.
L'archive ouverte pluridisciplinaire HAL, est destinée au dépôt et à la diffusion de documents scientifiques de niveau recherche, publiés ou non, émanant des établissements d'enseignement et de recherche français ou étrangers, des laboratoires publics ou privés. 


\title{
Cosmological constraints from CODEX galaxy clusters spectroscopically confirmed by SDSS-IV/SPIDERS DR16
}

\author{
J. Ider Chitham ${ }^{1 \star}$, J. Comparat ${ }^{1}$, A. Finoguenov ${ }^{2}$, N. Clerc $^{3}$, C. Kirkpatrick ${ }^{2}$, \\ S. Damsted ${ }^{2}$, A. Kukkola ${ }^{2}$, R. Capasso ${ }^{4}$, K. Nandra ${ }^{1}$, A. Merloni ${ }^{1}$, E. Bulbul ${ }^{1}$, \\ E. S. Rykoff ${ }^{5,6}$, D. P. Schneider ${ }^{7,8}$ and J. R. Brownstein ${ }^{9}$ \\ ${ }^{1}$ Max-Planck Institut für extraterrestrische Physik, Postfach 1312, D-85741 Garching bei München, Germany \\ ${ }^{2}$ Department of Physics, University of Helsinki, Gustaf Hällströmin katu 2a, FI-00014 Helsinki, Finland \\ ${ }^{3}$ IRAP, Université de Toulouse, CNRS, UPS, CNES, Toulouse, France \\ ${ }^{4}$ The Oskar Klein Centre, Department of Physics, Stockholm University, Albanova University Center, SE 10691 Stockholm, Sweden \\ ${ }^{5}$ Kavli Institute for Particle Astrophysics 83 Cosmology, PO Box 2450, Stanford University, Stanford, CA 94305, USA \\ ${ }^{6}$ SLAC National Accelerator Laboratory, Menlo Park, CA 94025, USA \\ ${ }^{7}$ Department of Astronomy and Astrophysics, The Pennsylvania State University, University Park, PA 16802 \\ ${ }^{8}$ Institute for Gravitation and the Cosmos, The Pennsylvania State University, University Park, PA 16802 \\ ${ }^{9}$ Department of Physics and Astronomy, University of Utah, 115 S. 1400 E., Salt Lake City, UT 84112, USA
}

Accepted 2020 September 22. Received 2020 September 8; in original form 2020 January 15

\begin{abstract}
This paper presents a cosmological analysis based on the properties of X-ray selected clusters of galaxies from the CODEX survey which have been spectroscopically followed up within the SPIDERS programme as part of the sixteenth data release (DR16) of SDSS-IV. The cosmological sub-sample contains a total of 691 clusters over an area of $5,350 \mathrm{deg}^{2}$ with newly measured optical properties provided by a reanalysis of the CODEX source catalogue using redMaPPer and the DESI Legacy Imaging Surveys (DR8). Optical richness is used as a proxy for the cluster mass, and the combination of X-ray, optical and spectroscopic information ensures that only confirmed virialised systems are considered. Clusters are binned in observed redshift, $\tilde{z} \in[0.1,0.6)$ and optical richness, $\tilde{\lambda} \in[25,148)$ and the number of clusters in each bin is modelled as a function of cosmological and richness-mass scaling relation parameters. A high-purity sub-sample of 691 clusters is used in the analysis and best fit cosmological parameters are found to be $\Omega_{m_{0}}=0.34_{-0.05}^{+0.09}$ and $\sigma_{8}=0.73_{-0.03}^{+0.03}$. The redshift evolution of the self-calibrated richness-mass relation is poorly constrained due to the systematic uncertainties associated with the X-ray component of the selection function (which assumes a fixed X-ray luminosity-mass relation with $h=0.7$ and $\Omega_{m_{0}}=0.30$ ). Repeating the analysis with the assumption of no redshift evolution is found to improve the consistency between both cosmological and scaling relation parameters with respect to recent galaxy cluster analyses in the literature.
\end{abstract}

Key words: cosmology: cosmological parameters $-\mathrm{X}$-rays: galaxies: clusters

\section{INTRODUCTION}

Galaxy clusters sit at the apex of cosmic hierarchy as virialised objects with masses ranging from approximately $10^{13} \mathrm{M}_{\odot}$ for groups, to $10^{15} \mathrm{M}_{\odot}$ for the richest clusters. Clusters originate from small amplitude density perturbations which deviated from the Hubble flow during the primordial Universe. These over-densities then relax, merge and coalesce, hierarchically to form progressively larger structures

^ jacobic@mpe.mpg.de through gravitational interactions (Peebles 1980; Bardeen et al. 1986). The abundance of clusters as a function of mass at different epochs (cluster mass function; Bahcall \& Cen 1993) depends on cosmological parameters and hence can be used to constrain them (for a review see Borgani \& Guzzo 2001; Allen et al. 2011; Weinberg et al. 2013; Pratt et al. 2019).

The cluster mass function is related to the dark matter halo mass function (HMF) as the largest component of cluster mass is non baryonic (Zwicky 1933; Navarro et al. 1997). The HMF depends on large scale structure formation 
(Sheth \& Tormen 2002; Zentner 2007; Tinker et al. 2008; Diemer \& Kravtsov 2015), and on the cosmological volume over which clusters are observed. These cosmological dependencies make cluster counts particularly sensitive to $\Omega_{m}$, the matter density of the Universe, and $\sigma_{8}$, the amplitude of the matter power spectrum.

Cluster count experiments (e.g. Vikhlinin et al. 2009b; Rozo et al. 2010; Mantz et al. 2015; Zu et al. 2014; Böhringer et al. 2014; Pacaud et al. 2018; Bocquet et al. 2019; Zubeldia \& Challinor 2019; Costanzi et al. 2019b; Kirby et al. 2019; Abbott et al. 2020a) provide an independent method to complement cosmological constraints from other probes, such as the cosmic microwave background (CMB, Planck Collaboration et al. 2020), Type Ia supernova (Riess et al. 1998; Perlmutter et al. 1999; Riess et al. 2018a; Jones et al. 2018; Scolnic et al. 2018), Cepheid variables (Riess et al. 2018b; Verde et al. 2019) baryonic acoustic oscillations (Eisenstein et al. 2005; Alam et al. 2017; Ata et al. 2018), redshift space distortions (Pezzotta et al. 2017; Zarrouk et al. 2018), and cosmic shear (Abazajian \& Dodelson 2003; Jullo et al. 2019; Abbott et al. 2020b).

Clusters exhibit well known observational signatures which enable them to be detected across several different wavelength domains. These include: (i) quiescent red sequence galaxies (Gladders \& Yee 2005; Koester et al. 2007b; Szabo et al. 2011) (ii) intra cluster light at optical and infrared wavelengths (Conroy et al. 2007; Burke et al. 2015) (iii) X-ray emission from the extended intra cluster gas (Kellogg et al. 1975; Böhringer et al. 2000; Vikhlinin et al. 2009a) and (iv) the spectral distortion of the cosmic microwave background via the Sunyaev-Zeldovich (SZ) effect (Sunyaev \& Zeldovich 1970, 1972; Birkinshaw 1999; Vanderlinde et al. 2010; Planck Collaboration et al. 2014).

In order to constrain cosmology, a sample assembled using one (or more) of these methods also requires estimates of cluster mass. Arguably the most direct and accurate massmeasurement technique is weak lensing (Murata et al. 2019; McClintock et al. 2019a; Phriksee et al. 2020; Umetsu 2020). In the case that lensing data are not available, cluster massproxies such as core-excised X-ray luminosity, integrated SZ effect signal, velocity dispersion, optical richness, etc. are used in combination with scaling relations to provide a link to the cluster mass and to the HMF.

$\mathrm{X}$-ray luminosity is one of the preferred mass proxies for cluster cosmology (Reiprich \& Böhringer 2002; Vikhlinin et al. 2009b; Mantz et al. 2015; Pacaud et al. 2018), however, optical richness is also a theoretically attractive mass proxy due to the small magnitude of the irreducible scatter (Old et al. 2018). Despite this, previous attempts to constrain cosmological parameters using richness (Bahcall et al. 2003; Gladders et al. 2007; Wen et al. 2010; Rozo et al. 2010; Tinker et al. 2012; Mana et al. 2013; Costanzi et al. 2019b) have been consistently dominated by the scatter due to noise in the measurement (Becker et al. 2007; Rykoff et al. 2012; Capasso et al. 2019).

The SPIDERS DR16 cluster sample (2,740 in total) is designed to alleviate the uncertainties induced by using photometric redshifts in cosmological analyses by ensuring each cluster is confirmed spectroscopically. The sample is constructed from CODEX; an X-ray selected cluster survey (based on ROSAT, Finoguenov et al. 2020), with optical information provided by running redMaPPer (Rykoff et al.
2014) in scanning-mode ${ }^{1}$ using SDSS DR8 photometry (Aihara et al. 2011). This sample has been reanalysed using photometry from DR8 of The Legacy Surveys (Dey et al. 2019) to produce a volume limited cluster catalogue with a lower scatter optical mass proxy than the original SPIDERS DR16 sample (4,448 in total).

This paper presents a cosmological interpretation of a number count analysis for a high-purity subset of SPIDERS DR16 clusters with a focus on constraining $\Omega_{m_{0}}, \sigma_{8}$ and the parameters of a self calibrated richness-mass scaling relation. All other parameters are fixed to their fiducial values assuming a flat $\Lambda \mathrm{CDM}$ cosmological model with a constant dark energy equation of state $w=-1$, a present day CMB temperature of 2.7255K (Fixsen 2009) and 3.046 effective neutrino species (Planck Collaboration et al. 2018) with $H_{0}=70 \mathrm{kms}^{-1} \mathrm{Mpc}^{-1}, \Omega_{b_{0}}=0.048$ and $n_{s}=0.96$.

Cluster masses and radii are consistently defined by a spherical over-density 200 times that of the critical density of the Universe (unless stated otherwise).

When referring to observables throughout this paper, the following notation is consistently used: a tilde above an observable $\tilde{O}$ symbolises an observed quantity; one without a tilde, $O$, refers to the true, unobserved quantity (e.g, of the underlying halo). Conditional probabilities such as the probability of $A$ given $B_{\mu}$ are denoted as $P(A \mid B)$.

The structure of the paper is organised as follows: Sect. 2 introduces the CODEX and SPIDERS cluster samples. Sect. 3 describes the modelling of the likelihood function. Sect. 4 summarises the constraints obtained for cosmological and scaling relation parameters using SPIDERS clusters. Sect. 5 provides an interpretation of the results and discusses potential sources of systematic uncertainties.

\section{DATA}

\subsection{X-ray selected cluster samples}

The SPectroscopic IDentification of eROSITA Sources observational program (SPIDERS Clerc et al. 2016) within SDSS-IV/eBOSS (Dawson et al. 2016; Blanton et al. 2017) aims to follow up X-ray detected clusters using the BOSS spectrographs (Smee et al. 2013) on the 2.5m SDSS telescope (Gunn et al. 2006). The SPIDERS cluster catalogue published as part of the sixteenth data release of the SDSS (DR16; Ahumada et al. 2020) consists of a set of spectroscopically validated galaxy clusters drawn from two X-ray galaxy cluster catalogues:

- CODEX, the COnstraining Dark Energy with X-rays sample (Finoguenov et al. 2020), constructed by reanalysing the publicly available data from the ROSAT All Sky Survey (RASS; Voges et al. 1999). It covers 10,800 deg ${ }^{2}$.

- XCLASS, the XMM CLuster Archive Super Survey catalogue constructed by reanalysing the publicly available $X M M$ data (Clerc et al. 2012; Sadibekova et al. 2014).

1 redMaPPer configured in scanning-mode uses prior knowledge of the positions of cluster centres, producing a sample that is primarily defined by the original selection method (e.g. X-ray or SZ Finoguenov et al. 2020; Bleem et al. 2020) (Rozo \& Rykoff 2014) rather than pure optical selection (searching for over-densities of red galaxies in the conventional cluster-finding mode). 


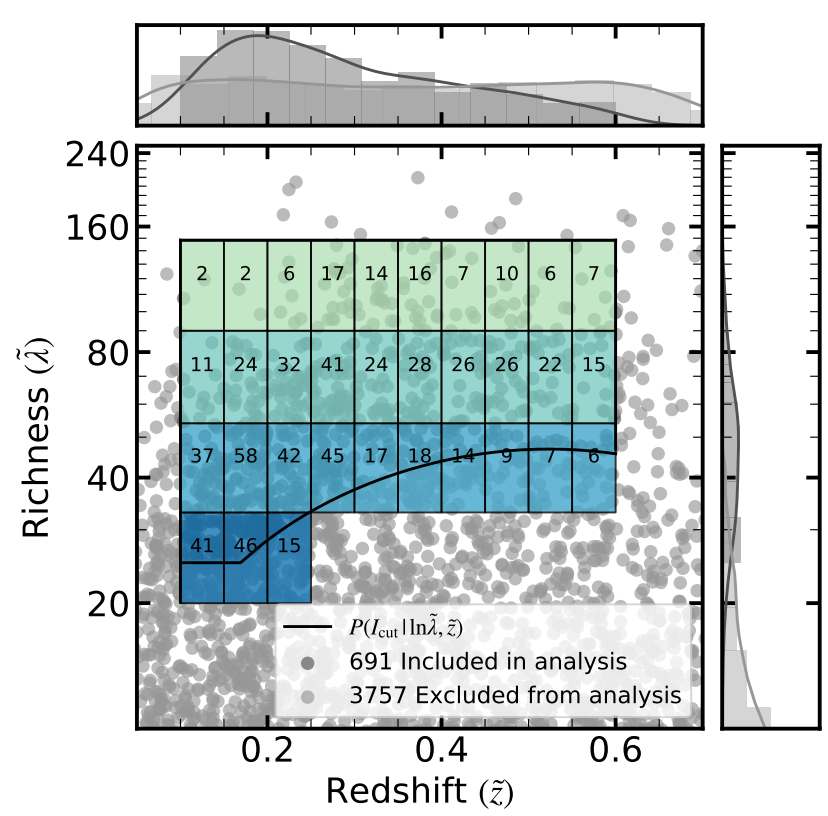

Figure 1. The volume limited cluster sample used in the cosmological analysis produced by analysing the original CODEX source catalogue with redMaPPer and The Legacy Surveys over the SPIDERS DR16 footprint. The solid black line represents the redshift dependent richness cut described by $P\left(I_{\text {cut }} \mid \ln \tilde{\lambda}, \tilde{z}\right)$ (Eq. 5). Boxes represent the bins used in this analysis, annotated with the respective clusters counts (post richness cut) and colours represent the richness bins highlighted in Fig. 2. The dark grey distributions illustrate clusters that are included in this analysis i.e. the 691 out of 4,448 clusters that fall within the coloured boxes and above the black line. The light grey distributions illustrate clusters excluded from the analysis.

Kirkpatrick et al. subm. and Clerc et al. (2020) thoroughly describe each component of the final DR16 SPIDERS catalogue in their respective companion papers.

Both catalogues are constructed by applying the redsequence Matched-filter Probabilistic Percolation clusterfinding algorithm (redMaPPer; Rykoff et al. 2014) to SDSSIII/BOSS DR8 imaging data (Fukugita et al. 1996; Aihara et al. 2011). This approach provides optical counterparts (over-densities of red galaxies) to the extended X-ray emission observed with the ROSAT/XMM observatories. Each optical counterpart is comprised of a set of potential member galaxies. The most probable members are observed spectroscopically (Clerc et al. 2016) and the sum of membership probabilities provides an estimate for the optical richness $\tilde{\lambda}_{\text {SDSS }}$ (estimated using the original SDSS derived photometric redshift, $\tilde{z}_{\lambda_{\text {SDSS }}}$; Rykoff et al. 2014). In this work, only the CODEX component of the SPIDERS cluster catalogue is considered in order to simplify the modelling of the overall selection function.

\subsection{DESI Legacy Imaging Surveys}

The DESI Legacy Imaging Surveys (hereafter The Legacy Surveys; Dey et al. 2019, 14,300 $\mathrm{deg}^{2}$ ) consist of The DECam Legacy Survey (DECaLS), The Beijing-Arizona Sky Survey (BASS) and The Mayall z-band Legacy Sur-

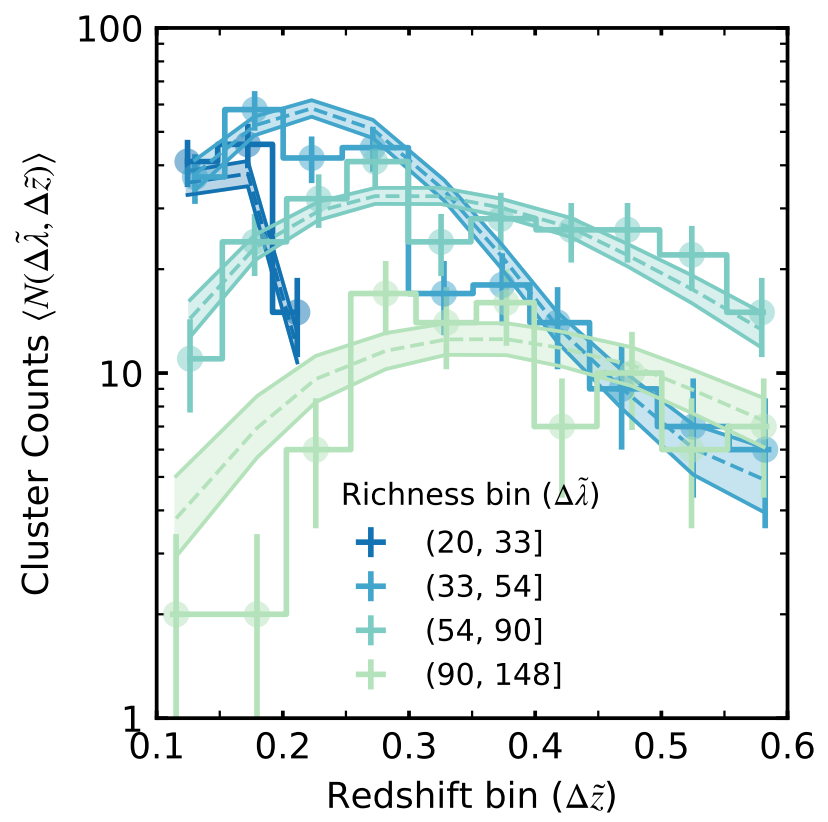

Figure 2. The abundance of SPIDERS clusters as a function in bins of observed redshift $\left(\Delta \tilde{z}_{j}\right)$ and richness $\left(\Delta \ln \tilde{\lambda}_{i}\right)$ where $\tilde{z} \in[0.1,0.6)$ and $\tilde{\lambda} \in[25,148)$. Steps represent the observed data, the width and height correspond to the size of the bin and the magnitude of the diagonal (Poisson) elements of the covariance matrix, respectively. The position of the vertical bars indicates the mean redshift in each bin. The shaded regions trace the expectation value provided by the model (with a normal prior on the intrinsic scatter Bleem et al. 2020), centred on the median, which corresponds to the best-fit cosmology. The lower and upper limit are similarly set by the $15 \%$ and $85 \%$ confidence intervals. These distributions are calculated directly from the stored expectation values of cluster counts for the MCMC chains used to create the contours shown in Fig. 5.

vey (MzLS). The eighth data release (DR8) of The Legacy Surveys also includes a variety of Dark Energy Camera (DECam) imaging from a variety of other sources such as the Dark Energy Survey (DES; The Dark Energy Survey Collaboration 2005, 5,000 $\mathrm{deg}^{2}$ ). These surveys span the entire BOSS (CODEX) footprint and therefore provide ancillary photometry in $g, r$ and $z$ bands that is at least 1-2 magnitudes deeper than the SDSS.

The availability of superior photometric data from DR8 of The Legacy Surveys thus allows one to obtain a lowerscatter optical mass-proxy $(\tilde{\lambda})$ than the original CODEX measurement of richness $\left(\tilde{\lambda}_{\text {SDSS }}\right)$. This is achieved by remeasuring richness while also estimating a new optical centre within $400 \mathrm{kpc}$ of the peak of the extended X-ray emission for each cluster using redMaPPer v6.6 configured in scanning-mode. The method used to apply redMaPPer to The Legacy Surveys is described in detail in Ider Chitham et al. in prep, although, the most cosmologically relevant information is also briefly summarised in Appendix A of this article.

The definition of richness is sensitive to the calibration procedure within redMaPPer. Influential factors include the 
initial spectroscopic galaxy training set, the number of iterations used to tune the models of red-sequence galaxies, the measurement of the background as well as the quality of the photometry (i.e. the level of optimisation with respect to colour and total flux). As almost all these things differ from the original CODEX redMaPPer processing with the SDSS, the remeasured richness based on The Legacy Surveys is found to be systematically lower than the original $\tilde{\lambda}_{\text {SDSS }}$ definition. The median ratio between the two definitions of richness is found to evolve with redshift as

$$
\tilde{\lambda}=\frac{\tilde{\lambda}_{\text {SDSS }}}{1.04+0.17 e^{5.4(\tilde{z}-0.36)}} \text {. }
$$

For SDSS redMaPPer selected clusters, richness extrapolation occurs when $\tilde{z} \gtrsim 0.36$ (Rykoff et al. 2014; Finoguenov et al. 2020). Such a boosting effect can result in richness being systematically up-scattered, hence the exponent in the denominator of Eq. 1.

\subsection{SPIDERS spectroscopy}

The SDSS-IV/SPIDERS spectroscopic data span 5,350deg ${ }^{2}$ out of the total 10,800 deg ${ }^{2}$ SDSS-III/BOSS footprint which is covered by CODEX. Within this area, the total number of spectroscopically validated and visually inspected clusters of galaxies with $\tilde{\lambda}_{\text {SDSS }}>10$ is 2,740 i.e. about 0.5 per square degree (Clerc et al. 2020, Kirkpatrick et al. subm.). For systems with $\tilde{\lambda}_{\text {SDSS }}>40$, a total of 920 out of 1047 were confirmed. The remaining 127 are either dubious candidates or high redshift systems $(\tilde{z}>0.7)$ lacking spectra.

To ensure the SPIDERS DR16 sample is as completeness as possible, spectroscopic redshifts are re-evaluated by matching previously observed spectroscopic galaxies with the newly defined member catalogue, which is generated when remeasuring richness with The Legacy Surveys (Sect. 2.2). The updated spectroscopic galaxies are then re-processed with the original SPIDERS automated cluster redshift pipeline by iteratively considering their distribution in phase space (Clerc et al. 2012, 2020, Kirkpatrick et al. subm.) and performing bootstrap re-sampling. A high-purity sub-sample of these clusters is used to constrain cosmology (Sect. 2.4) for which visual inspection is also carried out by a single inspector ${ }^{2}$.

\subsection{Cosmological cluster sub-sample}

The range over which observables (redshift and richness) are modelled directly impacts the accuracy and precision of the posterior distributions of the cosmological and richness-mass relation parameters derived in this work. Due to the tradeoff between accuracy and precision, one must optimise the size of the cluster sample to ensure that it is statistically significant enough to be sensitive to the parameters of the model while simultaneously excluding regions of the observable parameter space which are challenging to characterise.

27 bootstrap spectroscopic redshift estimates were adjusted in the visual inspection process after the pipeline raised flags regarding the convergence of the velocity clipping procedure. 4 spectroscopic central galaxies were assigned/adjusted in the visual inspection process due to mis-centring.
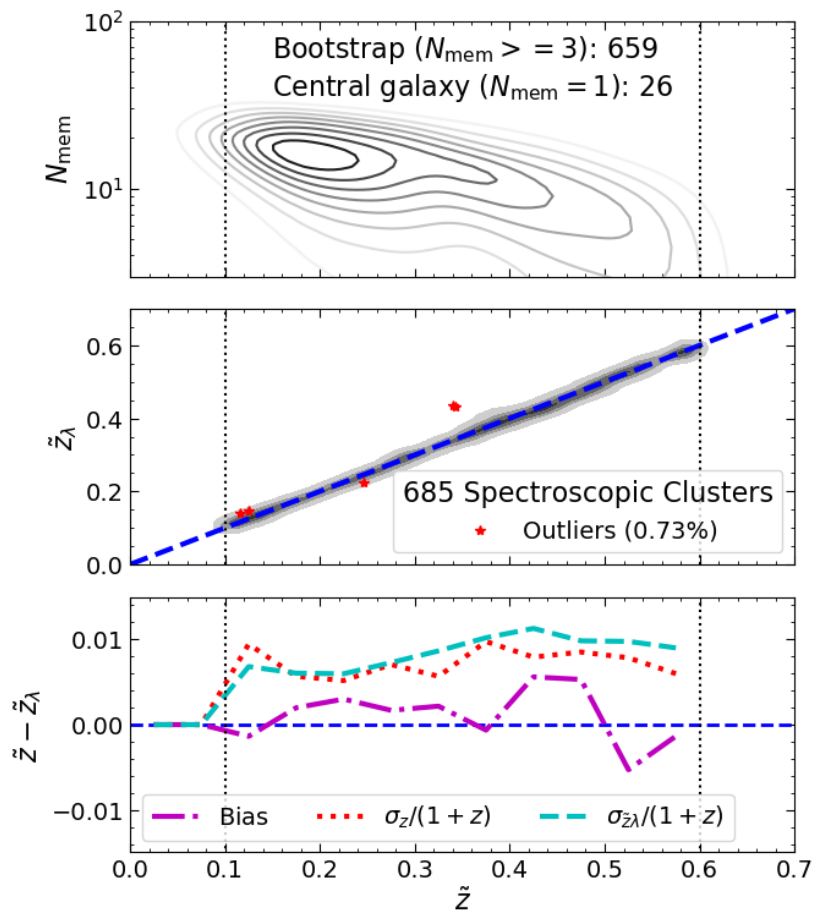

Figure 3. Cosmological cluster sub-sample after remeasuring spectroscopic redshifts for CODEX clusters using member galaxies selected as part of the reanalysis with The Legacy Surveys (Sect. 2.3). Upper panel: distribution of number of spectroscopic members, $N_{\text {mem }}$ as a function of redshift. Middle panel: spectroscopic redshift $\tilde{z}$ versus photometric redshift $\tilde{z}_{\lambda}$. Outliers are defined when the discrepancy between is more than three times the uncertainty on the photometric redshift $\left(\tilde{z}-\tilde{z_{\lambda}}>3 \sigma_{\tilde{z}_{\lambda}}\right)$. Lower panel, median quantities over each redshift bin used in this analysis (bin width of 0.05$)$ bias: $\left(\tilde{z}-\tilde{z_{\lambda}}\right)$, scatter: $\sigma_{z} /(1+\tilde{z})=$ $1.4862 \times\left(\left|\tilde{z}-\tilde{z_{\lambda}}\right|-\left(\tilde{z}-\tilde{z_{\lambda}}\right)\right) /(1+\tilde{z})$ and photometric redshift uncertainties: $\sigma_{\tilde{z}_{\lambda}} /(1+\tilde{z})$.

The latter is necessary to reduce the influence of unquantifiable systematic uncertainties. This section justifies the cuts applied to the observable parameter-space which define the cosmological cluster sub-sample used in this analysis. Fig. 1 illustrates the redshift and richness distribution of 4,448 CODEX clusters from The Legacy Surveys volumelimited $^{3}$ redMaPPer catalogue constructed over the SPIDERS DR16 footprint. The cosmological sub-sample of 691 clusters is shown by the points in the coloured boxes that are above the redshift dependant richness cut (Eq. 5) where the $\mathrm{X}$-ray selection function is most sensitive (Sect. 3.2). The abundance modelling for these clusters is described in the subsequent section (Sect. 3) and presented in Fig. 2.

659 clusters in the cosmological sub-sample have more than three spectroscopic member galaxies and an additional 26 systems have a single visually inspected central spectroscopic galaxy. This results in a mean of 15.7 spectroscopic members per cluster redshift, as shown top panel of Fig. 3, with statistical uncertainties of the order $\Delta_{z} /(1+\tilde{z}) \sim 7 \times 10^{-4}$.

3 For details of the volume limiting procedure, please refer to Sect. 3.2.2. 
The best available redshifts for the remaining 6 clusters are provided by photometric redshift estimates obtained during the richness re-measurement process (detailed in Appendix A).

The lower-limit of the observed-redshift range is set to 0.1 to avoid sub-optimal redMaPPer performance. Although this is less conservative than the limit of 0.2 used by Abbott et al. (2020a), it is justified by the fact that photometric redshifts of clusters in the cosmological sub-sample for $\tilde{z} \in[0.1,0.2)$ have extremely low-bias $(\sim 0.002)$ as well as the largest number of spectroscopic members per cluster $\left(N_{\text {mem }} \sim 20\right)$ as shown in Fig. 3 . The upper redshift limit is set to 0.6 to exclude clusters in the regime of spectroscopic incompleteness (Clerc et al. 2020).

Clusters with $\tilde{\lambda}<25$ are also excluded from this analysis. This hard limit is motivated by the fact that Abbott et al. (2020a) showed that excluding $\tilde{\lambda}_{\text {DES }}<30$ systems, systematically shifts the posterior distribution of $\Omega_{m_{0}}$ closer to that constrained by other cosmological probes (Beutler et al. 2011; Ross et al. 2015; Alam et al. 2017; Scolnic et al. 2018), indicating the presence of unknown effects related to low-richness systems. The numerical difference relative to the hard-limit imposed here is because the definition of richness for The Legacy Surveys is systematically lower $\left(\tilde{\lambda} \approx 0.87 \tilde{\lambda}_{\text {DES }}\right.$, Sect. 4.1). On top of this hard-limit at lowrichness, an additional conservative redshift-dependant richness cut (Sect. 3.2.3) is applied. This ensures an even smaller proportion of low-richness systems are considered above a redshift of 0.2 (Sect. 3.2.3 and Fig. 1). Clusters with $\tilde{\lambda}>148$ are also excluded from this analysis as they make up only $8 / 691$ when $\tilde{z} \in[0.1,0.6)$ and due to their rarity, a Gaussian likelihood model is not justified (Rozo et al. 2010).

Photometric clusters make up less than one percent of the cosmological cluster sub-sample and have a mean redshift and richness of 0.40 and 73.6 respectively. Of the 685 spectroscopic clusters, the fraction of systems with spectroscopic and photometric redshifts that differ by more than three times the estimated photometric uncertainty is also less than one percent (red outliers are shown in the middle panel of Fig. 3). The dashed purple and dotted red lines in the lower panel of Fig. 3 indicate that the photometric redshifts used in the cosmological cluster sub-sample are almost unbiased $\left(\tilde{z}-\tilde{z_{\lambda}} \sim 0.005\right)$, with low scatter $\left(\sigma_{z} /(1+\tilde{z}) \sim 0.01\right)$.

Conducting a cosmological analysis for the 2,740 spectroscopically validated clusters in the original SPIDERS DR16 sample with a revised mass-proxy from The Legacy Surveys would require the completeness of optical and spectroscopic observations as a function of cluster properties for the SDSS (Clerc et al. 2020) to be modelled. Given the sparsity and high quality of the redMaPPer photometric redshifts, the systematic effect impact of supplementing the spectroscopic sub-sample with 6 photometric clusters is negligible relative to the benefits of simplifying the overall selection function.

\section{MODEL}

This section presents the modelling used to interpret the cluster number counts in the observed data. The adopted form for the HMF is that of Tinker et al. 2008, with an accuracy of at the 10-20\% level, which is sufficient for this study.
Table 1. A tabular representation of Fig. 2, summarising cluster counts in bins of redshift and optical richness for the 691 SPIDERS DR16 galaxy clusters that comprise the cosmological cluster sub-sample outlined in Sect. 2.4. Parentheses contain values predicted by the best fit model (summarised in Table. 2) and statistical uncertainties taken from the diagonal (Poisson) elements of the covariance matrix.

\begin{tabular}{l|llll}
\hline$\Delta \tilde{z} / \Delta \tilde{\lambda}$ & $(25,33]$ & $(33,54]$ & $(54,90]$ & $(90,148]$ \\
\hline$(0.1,0.15]$ & $41(35 \pm 5)$ & $37(37 \pm 6)$ & $11(13 \pm 3)$ & $2(3 \pm 1)$ \\
$(0.15,0.2]$ & $46(37 \pm 6)$ & $58(52 \pm 7)$ & $24(22 \pm 4)$ & $2(6 \pm 2)$ \\
$(0.2,0.25]$ & $15(11 \pm 3)$ & $42(58 \pm 7)$ & $32(28 \pm 5)$ & $6(9 \pm 3)$ \\
$(0.25,0.3]$ & - & $45(52 \pm 7)$ & $41(32 \pm 5)$ & $17(11 \pm 3)$ \\
$(0.3,0.35]$ & - & $17(35 \pm 5)$ & $24(32 \pm 5)$ & $14(12 \pm 3)$ \\
$(0.35,0.4]$ & - & $18(22 \pm 4)$ & $28(30 \pm 5)$ & $16(12 \pm 3)$ \\
$(0.4,0.45]$ & - & $14(14 \pm 3)$ & $26(26 \pm 5)$ & $7(11 \pm 3)$ \\
$(0.45,0.5]$ & - & $9(9 \pm 3)$ & $26(22 \pm 4)$ & $10(10 \pm 3)$ \\
$(0.5,0.55]$ & - & $7(6 \pm 2)$ & $22(18 \pm 4)$ & $6(9 \pm 3)$ \\
$(0.55,0.6]$ & - & $6(5 \pm 2)$ & $15(13 \pm 3)$ & $7(7 \pm 2)$ \\
\hline
\end{tabular}

This HMF is mapped to the richness observable through a scaling relation (Sect. 3.1). The selection function (Sect. 3.2) is then convolved to obtain a prediction of the number counts in the same binning scheme as the observations (Sect. 3.3). Finally, cosmological and scaling relations parameters are jointly fit by comparing the model to the data assuming a Poisson likelihood (Sect. 3.4). To ensure the likelihood function and code works as expected, a series of validation tests have also been carried out as detailed in Appendix B.

\subsection{Scaling Relation}

The scaling relation relates the true halo mass $(\mu=\ln M)$ to the true richness $(\lambda)$ as a function of true redshift $(z)$. The natural logarithm of the expected richness is given by

$\langle\ln \lambda(\mu, z)\rangle=A+B\left(\mu-\mu_{\text {pivot }}\right)+B_{z} \ln \left(\frac{1+z}{1+z_{\text {pivot }}}\right)$.

Here, $z_{\text {pivot }}$ and $\mu_{\text {pivot }}$ are pivot values of redshift and the mass which equate to 0.263 and the natural logarithm of $3.08 \times 10^{14} \mathrm{M}_{\odot} h^{-1}$. For convenience, these values are chosen to be the median redshift and mass for the cosmological cluster sub-sample (Sect. 2.4) when assuming masses inferred from X-ray luminosity (Finoguenov et al. 2020). The remaining quantities are dimensionless free parameters: $A$ is the expectation value at the pivot point $\left\langle\ln \lambda\left(\mu_{\text {pivot }}, z_{\text {pivot }}\right)\right\rangle, B_{\mu}$ is the coefficient of halo mass dependence, $B_{z}$ is the linear coefficient of redshift evolution.

The overall variance on richness is modelled as

$\sigma^{2}(\mu, z)=\sigma_{\text {int }}^{2}(\mu, z)+\sigma_{\text {noise }}^{2}(\ln \lambda, z)=\sigma_{\text {int }}^{2}+\lambda^{-1}$.

where $\sigma_{\text {int }}$ is the intrinsic scatter about the expected richness, which is assumed to be independent of halo mass and true redshift (e.g. Capasso et al. 2019), and $1 / \sqrt{\lambda}$ is the Poisson noise on the true richness from summing the membership probabilities of galaxies.

The priors for the richness-mass scaling relation parameters are summarised in Table. 2 and initialised to the bestfit values of Capasso et al. (2019). The prior for the normalisation of the richness-mass relation is set to the natural logarithm of the observed-richness range used in this analysis i.e. $A \in[3.0,5.0]$. As richness scales with mass, 
the prior of mass dependency is set to enforce a positive trend with a wide range of possible values $B_{\mu} \in[0.0,10.0)$ (e.g. Kiiveri et al. subm.). The prior for the redshift evolution is set to $B_{z} \in[-5.0,5.0]$ (Abbott et al. 2020a) and several different priors are considered for the intrinsic scatter in Sect. 4.3. These intrinsic scatter priors include that of the recent DES analysis, $\sigma_{\text {int }} \in[0.1,0.5]$ (justified in Appendix B of Abbott et al. 2020a), a more restrictive variant of this, $\sigma_{\text {int }} \in[0.1,0.3]$, as well a normal prior centred on the best fit value from Bleem et al. (2020). In addition to these basic priors, restrictions are also made to ensure only physically plausible parameter combinations are considered i.e. $\langle\ln \lambda(\mu, z)\rangle>1$ for all values of true mass and true redshift.

\subsection{Selection function}

The cosmological sub-sample of SPIDERS DR16 cluster is obtained via the aggregation of multiple selection processes: primary X-ray selection, followed by secondary optical selection. These components of the total selection are described below. The variable $I$ is used to denote selection and each component of the total selection $I_{\text {tot }}$ has a representative subscript e.g. $I_{\mathrm{X}}$ indicates X-ray selection, $I_{\text {Legacy }}$ represents the selection due to applying redMaPPer to The Legacy Surveys and $I_{\text {cut }}$ describes the redshift dependant richness cut applied to the X-ray selected clusters.

\subsubsection{X-ray selection function}

The form of the X-ray selection function is explained in detail in the CODEX catalogue paper (Finoguenov et al. 2020). The selection term (Eq. 9 of Finoguenov et al. 2020), denoted here as $P\left(I_{\mathrm{X}} \mid \mu, z, v\right)$ provides the probability of cluster detection as a function of true quantities $\mu, z$ as well as $v$ (Eq. 4). $v$ is defined as the fraction between the deviation of the true richness from its expectation value (Eq. 2) and the intrinsic scatter $\left(\sigma_{\text {int }}\right)$ :

$$
v \equiv \frac{\ln \lambda-\langle\ln \lambda(\mu, z)\rangle}{\sigma_{\text {int }}}, \quad \frac{\mathrm{d} v}{\mathrm{~d} \ln \lambda}=\frac{1}{\sigma_{\text {int }}} .
$$

The definition of $P\left(I_{\mathrm{X}} \mid \mu, z, v\right)$ includes a volume correction due to the survey mask described in Sect. 2.2. $P\left(I_{\mathrm{X}} \mid \mu, z, v\right)$ also has a small cosmological dependence via the luminosity distance and its impact increases with redshift. In this analysis, $P\left(I_{\mathrm{X}} \mid \mu, z, v\right)$ is evaluated at the fiducial cosmology $\Omega_{m_{0}}=0.30$ and $h=0.7$. This probability distribution is illustrated in Fig. 4 and systematic uncertainties due to this approximation are estimated in Sect. 5.1.4.

\subsection{2 redMaPPer selection function}

The first optical selection term, $P\left(I_{\text {Legacy }} \mid \ln \tilde{\lambda}, \tilde{z}\right)$, accounts for any differences in the area coverage and masking between in the original SDSS based SPIDERS footprint and the one based on The Legacy Surveys used in this analysis. It also considers the fact that the sample is locally volume limited (Rykoff et al. 2016; Abbott et al. 2020a; Bleem et al. 2020, discussed in Appendix A) in order to reduce the Eddington (1913) bias. This simply means that the depth of the survey is used to estimate the maximum redshift at which galaxies at a z-band luminosity cut of $L *<0.2$ can be observed in The
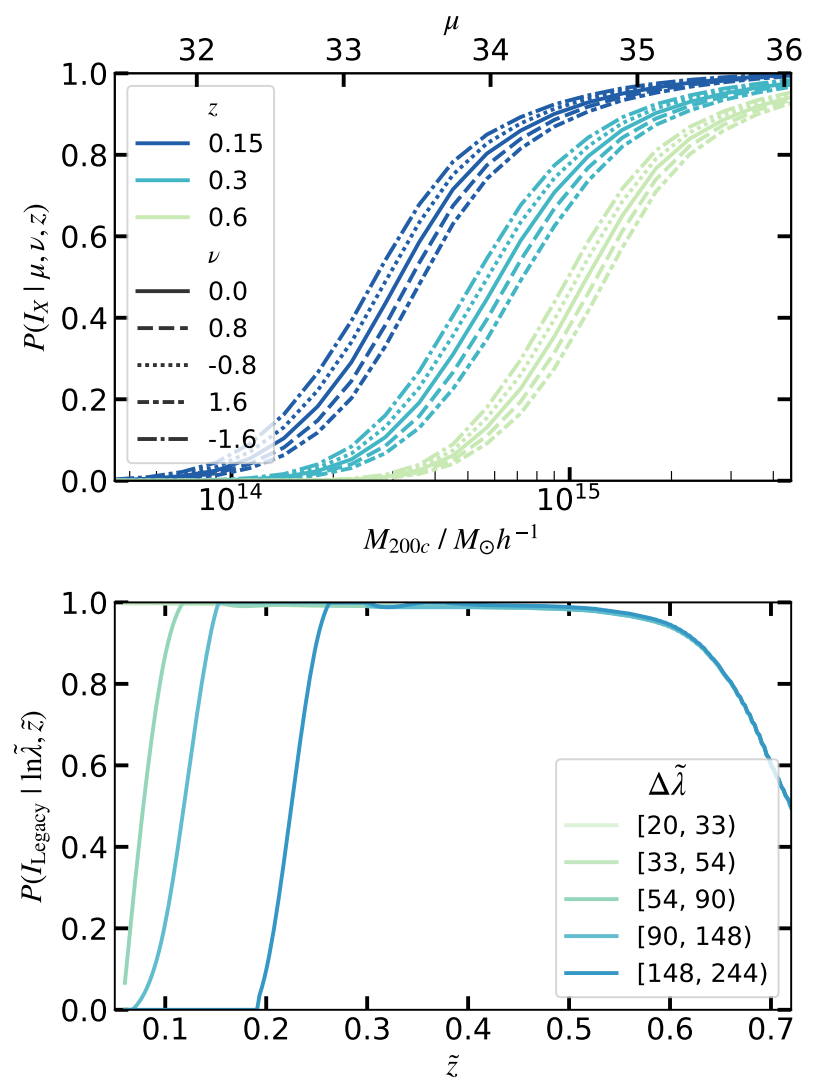

Figure 4. Upper panel: CODEX X-ray selection function, $P\left(I_{\mathrm{X}} \mid \mu, z, v\right)$ (defined by Eq. 9 of Finoguenov et al. 2020, for the SPIDERS DR16 footprint over The Legacy Surveys). Solid lines show the probability of detection as a function of mass $\left(\mu=\ln M_{200 c}\right)$ and true redshift (colour coded) when the expected richness of the richness-mass relation is equal to the true richness (i.e. $v=0$, see Eq. 4). The dotted and dashed variants of the solid lines illustrate how the X-ray selection function changes when the difference between the expected richness and true richness is equal to $-1.6,-0.8,0.8$ or 1.6 times the intrinsic scatter of the richnessmass relation. Lower panel: $P\left(I_{\text {Legacy }} \mid \ln \tilde{\lambda}, \tilde{z}\right)$, the optical selection function describing the completeness of the volume-limited redMaPPer catalogue of CODEX clusters with The Legacy Surveys over the SPIDERS DR16 footprint (Sect. 3.2.2). This considers the magnitude/luminosity cut $(L *<0.2)$, minimum richness $(\tilde{\lambda}>5)$ and maximum masking fraction $(0.2)$.

Legacy Surveys (at the $10 \sigma$ confidence level) as a function of position on the sky. Clusters which exceed this maximum redshift and the maximum masking fraction $(20 \%$ in this case) are excluded from the volume-limited catalogue. The $P\left(I_{\text {Legacy }} \mid \ln \tilde{\lambda}, \tilde{z}\right)$ distribution, illustrated in the lower panel of Fig. 4, is generated by redMaPPer for each redshift and richness bin using weighted randoms points (for more detail, please refer to Sect. 3.6 of Rykoff et al. 2016).

\subsubsection{Redshift dependant richness cut}

The second optical selection, $P\left(I_{\text {cut }} \mid \ln \tilde{\lambda}, \tilde{z}\right)$, describes a redshift dependent cut in observed-richness (Eq. 5) introduced to minimise contamination from spurious X-ray sources 
while retaining as many true systems as possible (also known as optical cleaning e.g. Klein et al. 2018, 2019; Grandis et al. 2020). This is shown by the solid black boundary which slices through the coloured bins in Fig. 1.

$P\left(I_{\text {cut }} \mid \ln \tilde{\lambda}, \tilde{z}\right)=\theta\left(25 \tilde{\lambda}_{\operatorname{SDSS}}(\tilde{z} / 0.15)^{0.8}\right)$

Here, $\theta$ is the Heaviside step function. The argument ${ }^{4}$ corresponds to the threshold above which the X-ray detection of CODEX cluster exceeds a probability of $>50 \%$ $\left(P\left(I_{\mathrm{X}} \mid \mu, z, v\right)>0.5\right)$ under the assumption of the fiducial richness-mass relation (Capasso et al. 2019). Such a conservative cut is motivated by the impact of the systematic uncertainties of the fixed X-ray luminosity-mass relation on the cosmological and richness-mass scaling relation parameters constrained in this work (discussed in Sect. 5.1.4). Although applying such a cut significantly reduces the total number of systems, and therefore the precision of the constrained parameters, the remaining sub-sample is extremely high-purity with a contamination level of $<5 \%$.

\subsubsection{Total selection function}

The probability of observing a cluster with $\tilde{\lambda}$ (given mass and redshift) is obtained by integrating the product of the overall selection, $P\left(I_{\text {tot }} \mid \tilde{z}, \ln \tilde{\lambda}, \mu, z, v\right)$, with a log-normal distribution of observed richness with a mean with given by the true richness, $P(\ln \tilde{\lambda} \mid \ln \lambda, \mu, z)$. The resulting term (Eq. 6) essentially provides the link between the richness-mass relation and the halo mass function (see definition in Sect. 3.3):

$$
\begin{aligned}
P(\ln \tilde{\lambda} \mid \mu, z)=\int_{\langle\ln \lambda(\mu, z)\rangle-4 \sigma_{\text {int }}}^{\langle\ln \lambda(\mu, z)\rangle+4 \sigma_{\text {int }}} \mathrm{d} \ln \lambda & P(\ln \tilde{\lambda} \mid \ln \lambda, \mu, z) \\
\cdot & P\left(I_{\text {tot }} \mid \tilde{z}, \ln \tilde{\lambda}, \mu, z, v\right) .
\end{aligned}
$$

Here, $P(\ln \tilde{\lambda} \mid \ln \lambda, \mu, z)=\mathcal{N}(\ln \tilde{\lambda}, \ln \lambda, \sqrt{\lambda})$, i.e. a log-normal distribution of observed richness, centred on the true value of richness with scatter described by the noise term in Eq. 3. The limits of the integral are determined by the minimum and maximum deviation from the fiducial richnessrelation Eqs. 2 and 3 considered during the construction of the X-ray selection function. This can be simplified as an expression in terms of $v$ (introduced in Eq. 4) by changing limits and introducing an additional term $P(v)=\mathcal{N}(v, 0,1)$. Expanding the total selection, $P\left(I_{\text {tot }} \mid \tilde{z}, \ln \tilde{\lambda}, \mu, z, v\right)=P\left(I_{X}, I_{\text {Legacy }}, I_{\text {cut }} \mid \tilde{z}, \ln \tilde{\lambda}, \mu, z, v\right)$, into its separate components then gives

$$
\begin{array}{r}
P(\ln \tilde{\lambda} \mid \mu, z)=\int_{-4}^{+4} \mathrm{~d} v P(v) \cdot P(\ln \tilde{\lambda} \mid \ln \lambda(\mu, z, v), z) \\
\cdot P\left(I_{\mathrm{X}} \mid \mu, z, v\right) \\
\cdot P\left(I_{\text {Legacy }} \mid \ln \tilde{\lambda}, \tilde{z}\right) \\
\cdot P\left(I_{\text {cut }} \mid \ln \tilde{\lambda}, \tilde{z}\right) .
\end{array}
$$

${ }^{4}$ Please note that Eq. 5 is in terms of the original SDSS-based CODEX richness $\left(\tilde{\lambda}_{\text {SDSS }}\right)$ and is necessarily converted to The Legacy Surveys definition of richness using Eq. 1.

5 Using a redshift dependent richness cut which corresponds to the $10 \%$ sensitivity level of the CODEX survey is estimated to result in approximately $5 \%$ of the selected X-ray sources being non-clusters (Finoguenov et al. 2020), therefore an equivalent cut at the $50 \%$ sensitivity level is expected to reduce this significantly.

\subsection{Expected number counts}

The expectation value of the number of galaxy clusters per bin $N\left(\Delta \ln \tilde{\lambda}_{i}, \Delta \tilde{z}_{j}\right)$ is given by Eq. 8 , where the integration limits are consistently reduced to the subset of the parameter-space where the X-ray selection function is sensitive (Sect. 3.2) i.e. $z \in[0.05,0.75], v \in[-4,4]$ and $\mu \in$ $[30.7,35.3]$ (or equivalently $\log _{10}\left(M_{200 \mathrm{c}}\right) \in[13.5,15.5] \mathrm{M}_{\odot}$ ).

$$
\begin{aligned}
\left\langle N\left(\Delta \ln \tilde{\lambda}_{i}, \Delta \tilde{z}_{j}\right)\right\rangle= & \int_{0.05}^{0.75} \mathrm{~d} z \frac{\mathrm{d} V(z)}{\mathrm{d} z} \\
& \cdot\left\langle n\left(\Delta \ln \tilde{\lambda}_{i}, z\right)\right\rangle \int_{\Delta \tilde{z}_{j}} \mathrm{~d} \tilde{z} P\left(\tilde{z} \mid z, \Delta \ln \tilde{\lambda}_{i}\right),
\end{aligned}
$$

Here $\mathrm{d} V(z) / \mathrm{d} z$ is differential comoving volume element for a flat universe (Hogg 1999) and $P\left(\tilde{z} \mid z, \Delta \ln \tilde{\lambda}_{i}\right)=$ $\mathcal{N}\left(z,\left\langle\tilde{z}_{i j}\right\rangle, \sigma_{\tilde{z}_{i j}}\right)$, i.e. a normal distribution accounting for uncertainties on the mean observed redshift in each bin ${ }^{6}$. The quantity $\left\langle n\left(\Delta \ln \tilde{\lambda}_{i}, z\right)\right\rangle$ is the expected comoving number density of halos in the richness bin.

$\left\langle n\left(\Delta \ln \tilde{\lambda}_{i}, z\right)\right\rangle=\int_{30.7}^{35.3} \mathrm{~d} \mu \frac{\mathrm{d} n(\mu, z)}{\mathrm{d} \mu} \int_{\Delta \ln \tilde{\lambda}_{i}} \mathrm{~d} \ln \tilde{\lambda} P(\ln \tilde{\lambda} \mid \mu, z)$.

This calculation depends on the differential HMF defined in terms of $\mu$; the natural logarithm of the halo mass within a radius, $R$, as well as $\sigma(\mu)$; the RMS fluctuation of the smoothed matter density field. Here $f(\sigma)$ is the Tinker et al. (2008) multiplicity function ${ }^{7}$.

$\frac{\mathrm{d} n(\mu, z)}{\mathrm{d} \mu}=\frac{3}{4 \pi R^{3}(\mu, z)} \frac{\mathrm{d} \ln \sigma(\mu)^{-1}}{\mathrm{~d} \mu} f(\sigma(\mu), z)$.

A hard prior on $\Omega_{m_{0}}$ is given by the mean spherical overdensity relative to the range used to calibrate the Tinker et al. 2008 HMF. When using a critical over-density definition of mass this prior translates as $\Delta_{m}(z) \equiv \Delta_{c} \Omega_{m}(z) \in$ $(200,3200)$ (Bocquet et al. 2019).

\subsection{Parameter fitting \& likelihood function}

Cosmological and scaling relation parameters, contained in the vector $\boldsymbol{\theta}$, span a relatively high number of dimensions, therefore in order to heuristically fit a model to the binned data it is necessary to utilise a Monte Carlo Markov Chain (MCMC) sampler. The chosen sampler used in this work is emcee (Foreman-Mackey et al. 2013). The natural logarithm of the generic likelihood function has the following form

$\ln \mathcal{L}(\boldsymbol{N} \mid \boldsymbol{\theta}) \propto-\frac{1}{2}\left[(\boldsymbol{N}-\langle\boldsymbol{N}(\boldsymbol{\theta})\rangle)^{T} \boldsymbol{C}^{-1}(\boldsymbol{N}-\langle\boldsymbol{N}(\boldsymbol{\theta})\rangle)\right]$,

where $\boldsymbol{N}$ and $\langle\boldsymbol{N}(\boldsymbol{\theta})\rangle$ represent matrices of cluster counts in the data and expectation values provided by the model, respectively. Each element of the matrix represent a bin of $\Delta \ln \tilde{\lambda}_{i}$ and $\Delta \tilde{z}_{j}$, and $\boldsymbol{C}$ is the covariance matrix. The Poisson contribution to the covariance matrix is simply given by a diagonal matrix of the expectation value of counts in each bin $\boldsymbol{C}^{\text {Poisson }}=\delta_{\mathrm{ij}}\langle\boldsymbol{N}\rangle_{i}$, where $\delta_{\mathrm{ij}}$ represents the Kronecker delta function. The contribution due to sample variance $(\mathrm{Hu}$ \& Kravtsov 2003) is ignored as it is subdominant in the

\footnotetext{
6 The PDF of observed redshift given the true redshift is modelled as a Dirac delta as justified in Sect. 5.1.2

7 Eq. 10 is calculated using the colossus package (Diemer 2018).
} 
Table 2. Summary of measured parameters and their initial priors. The fiducial column describes the starting value of the Markov chains in the parameter space. In this column, cosmological parameters are those used to pre-compute the X-ray selection function described in Sect. 3.2.1 and scaling relation parameters are initialised to their fiducial values (Capasso et al. 2019). Posterior distributions correspond to the twenty-fourth percentile about the median as summarised by the diagonal elements of Fig. 5 .

\begin{tabular}{cccc}
\hline \hline Parameter & Fiducial & Prior & Posterior \\
\hline$\Omega_{m_{0}}$ & 0.30 & $\in 0.1,0.8]$ & $0.34_{-0.05}^{+0.09}$ \\
$\sigma_{8}$ & 0.780 & $\in[0.4,1.2]$ & $0.73_{-0.03}^{+0.03}$ \\
$A$ & $\sim 3.68$ & $\in[3.0,5.0]$ & $3.96_{-0.30}^{+0.15}$ \\
$B_{\mu}$ & 0.98 & $\in[0.0,10.0]$ & $1.07_{-0.20}^{+0.22}$ \\
$B_{z}$ & -1.08 & $\in[-5.0,5.0]$ & $1.42_{-0.69}^{+0.72}$ \\
$\sigma_{\text {int }}$ & 0.22 & $\sim \mathcal{N}(0.23,0.16)$ & $0.29_{-0.19}^{+0.18}$ \\
\hline
\end{tabular}

$\Omega_{m_{0}}$ represents the mean matter density of the universe at redshift zero.

$\sigma_{8}$ is the amplitude of the matter power spectrum.

$A$ is $\langle\ln \lambda(\mu, z)\rangle$ at pivot mass scale and pivot redshift.

$B_{\mu}$ is the coefficient of halo mass dependence in $\langle\ln \lambda(\mu, z)\rangle$.

$B_{z}$ is the coefficient of linear redshift dependence in $\langle\ln \lambda(\mu, z)\rangle$.

$\sigma_{\text {int }}$ is the intrinsic scatter of the richness-mass relation.

regime of high mass (richness), therefore a Poisson likelihood is a good approximation given that the majority of lowrichness systems are removed by the conservative redshift dependant richness cut Eq. 5.

\subsection{Binning strategy}

In order to determine the bin-size for the fiducial SPIDERS DR16 cosmological analysis, the experiment has been repeated over a grid of linear redshift bins $\left|\Delta \tilde{z}_{j}\right| \in\{0.1,0.05,0.025,0.0125\}$ and logarithmic richness bins $\left|\Delta \ln \tilde{\lambda}_{i}\right| \in\{0.625,0.5,0.3125\}$ for the cosmological cluster subsample (Sect. 2.4, as well as the validation dataset detailed in Appendix B). The aim being to find the binning scheme that minimises uncertainties from Poisson noise that otherwise limit the minimum width of the posterior distributions of both cosmological and scaling relation parameters (via Eq. 11). The precision for each run is given by the volume of the parameter space spanned by the Markov chains at the point of convergence. Using the sum of the squares of the fractional uncertainties on all the constrained parameters as an approximate performance indicator, the optimal (fiducial) configuration was to be $\left|\Delta \tilde{z}_{j}\right|=0.05,\left|\Delta \ln \tilde{\lambda}_{i}\right|=0.5$, corresponding to ten bins of observed redshift and 4 bins of observed richness.

\section{RESULTS}

The best fit model (Sect. 3.3) to the abundance data (Sect. 2.4) is summarised visually in Fig. 2 and numerically in Table. 1. The corresponding posterior distributions of measured parameters are shown in Fig. 5 along with contours indicating the 68 and $95 \%$ confidence levels. The fiducial, prior and posterior values of these parameters are also summarised in Table. 2, with best fit values found to be $\Omega_{m_{0}}=0.34_{-0.05}^{+0.09}, \sigma_{8}=0.73_{-0.03}^{+0.03}, A=3.96_{-0.30}^{+0.15}, B=1.07_{-0.20}^{+0.22}$, $B_{z}=1.42_{-0.69}^{+0.72}$ and $\sigma_{\text {int }}=0.29_{-0.19}^{+0.18}$
The pipeline used to constrain these parameters is validated in Appendix B using a synthetic volume-limited catalogue of CODEX clusters over the SPIDERS DR16 area (Comparat et al. 2020) after generating richness via the Bleem et al. (2020) relation over the same richness and redshift range as the observed cosmological cluster sub-sample as summarised in Figs. B1 and B2. The same likelihood model described in Sect. 3 is implemented on the simulated data and all input cosmological and richness-mass relation parameters are comfortably recovered within one standard deviation as shown by Figs. B3 and B4 and displayed numerically in Table. B1.

The following subsections attempt to interpret the parameters constrained from the observed cluster catalogue. This includes a comparison of the richness-mass relation to the most recent calibration experiments and the measured cosmological parameters to constraints obtained by other recent cluster cosmology experiments that cover a similar volume.

\subsection{Richness-mass relation constraints}

The richness-mass relation corresponding to the best fit parameters at the pivot mass, $3.08 \times 10^{14} \mathrm{M}_{\odot} h^{-1}$ and redshift 0.263 is shown in Fig. 6 along side several recently calibrated richness-relations from the literature:

- Capasso et al. (2019): derived from 428 SPIDERS clusters using dynamical mass calibration and the original SDSS based measurements of richness for CODEX clusters. The redshift range, $\tilde{z} \in[0.1,0.7)$, is similar to the one presented in this paper although the richness range is much larger with $\tilde{\lambda}_{\text {SDSS }}>20$. Clusters are selected via the minimum number of spectroscopic members per cluster $N_{\text {mem }}>10$. The pivot mass and redshift are $M_{200 c}=3 \times 10^{14} \mathrm{M}_{\odot}$ and 0.18 respectively. The version of relation shown in Fig. 6 also accounts for correlated scatter between richness and X-ray luminosity using the CODEX selection function (Finoguenov et al. 2020). For comparative purposes, observed richness is converted to The Legacy Surveys definition using Eq. 1.

- Bleem et al. (2020): calibrated using the Joint SPTredMaPPer Cluster Sample from the 2,770 $\mathrm{deg}^{2}$ SPTPol Extended Cluster Survey and DES redMaPPer catalogue. This relation has a pivot redshift of 0.6 and pivot mass of $M_{500 \mathrm{c}}=3 \times 10^{14} \mathrm{M}_{\odot} h^{-1}$. Masses are converted from $M_{500 \mathrm{c}}$ to $M_{200 \mathrm{c}}$ using a Child et al. (2018) halo concentration model and a NFW profile (Navarro et al. 1997). The conversion between DES and SDSS richness is carried out using $\tilde{\lambda}_{\text {SDSS }} \approx 0.93 \tilde{\lambda}_{\text {DES }}$ (McClintock et al. 2019a) in combination with Eq. 1. As DES photometric data are complete, no redshift scaling is applied to the richness value when applying the conversion between SDSS and The Legacy Surveys definitions. This leads to $\tilde{\lambda} \approx 0.87 \tilde{\lambda}_{\text {DES }}$.

At the pivot redshift (0.263), the normalisation and mass evolution of the best fit relation is remarkably similar to that of Capasso et al. (2019) and Bleem et al. (2020) although at the pivot mass $\left(3.08 \times 10^{14} \mathrm{M}_{\odot} h^{-1}\right)$ the redshift trend is vastly different. This work indicates a moderate, positive redshift evolution rather than predicting richness which decreases with redshift (Capasso et al. 2019), or little to no redshift evolution (Bleem et al. 2020). It is likely that the difference in the selection methods used to construct 


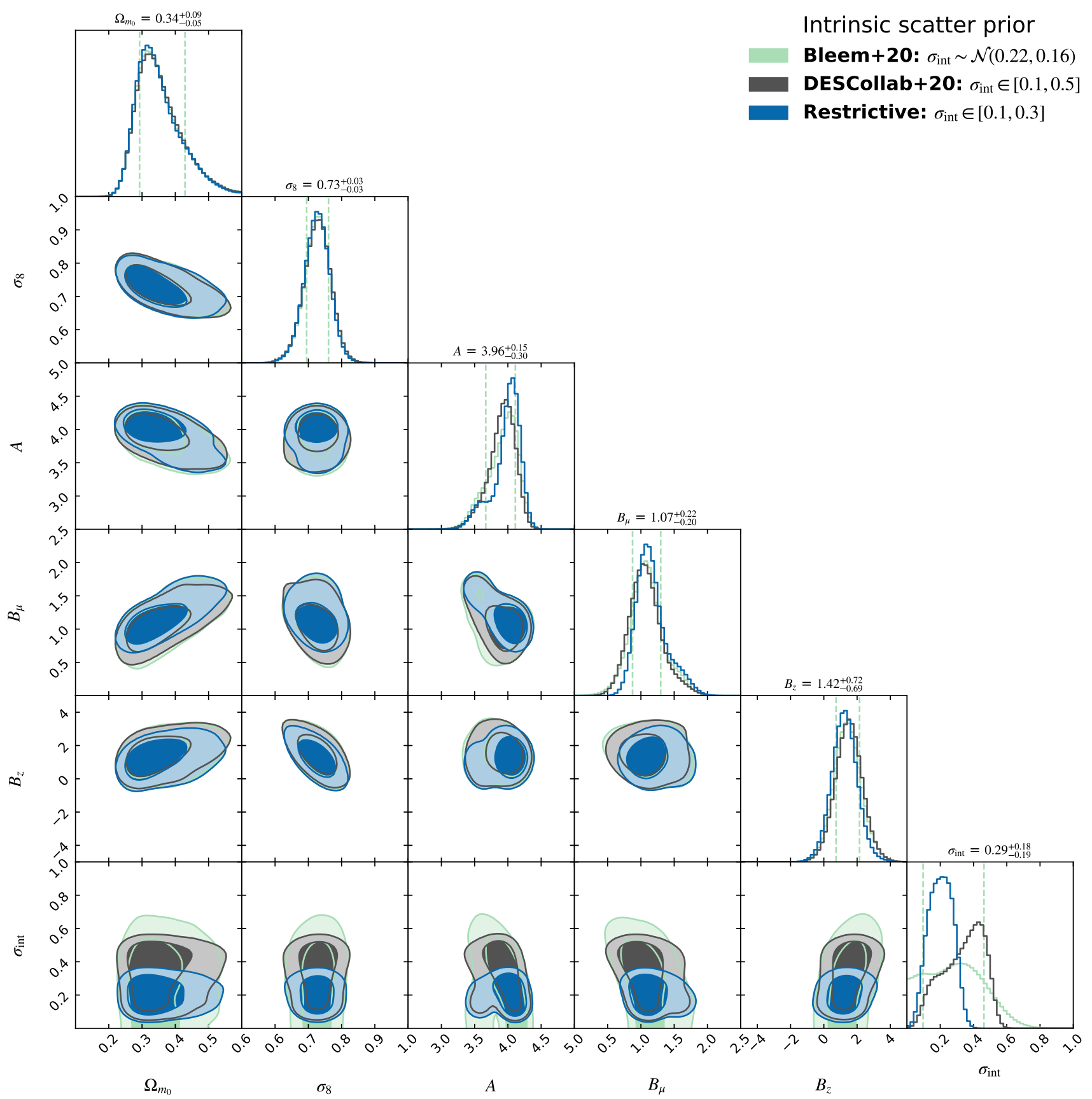

Figure 5. Cosmological parameters and scaling relation parameter constraints for SPIDERS DR16 as summarised in Table. 2. Contours depict the $68 \%$ and $95 \%$ confidence levels where posterior distributions are obtained using the likelihood function outlined in Sect. 3.4. The impact of the intrinsic scatter prior is shown by the different coloured posteriors (see legend).

each sample contributes to the discrepancy in the redshift trends, although it is currently not possible to draw any strong conclusions due to the lack of statistical significance on the $B_{z}$ parameter constraints. The wide posterior distribution of $B_{z}$ in Fig. 5 is indicative of $\sim 50 \%$ measurement uncertainties with systematic uncertainties also estimated to be $\gtrsim 50 \%$ (Sect. 5.1.4). The degeneracy between $B_{z}$ and cosmological parameters suggests that its value is in fact consistent with the derived cosmology (positive redshift evolution corresponds to high- $\Omega_{m_{0}}$ and low- $\sigma_{8}$ relative to a Planck-like cosmology). Repeating the experiment with a fixed value of $B_{z}=0$, confirms this by resulting in cosmological parameters that are more closely aligned with the median value of of recent cluster analyses shown in Fig. 7 (i.e. it increases the degree of similarity with respect to the cosmological constraints of Mantz et al. 2015; Zubeldia \& Challinor 2019; Bocquet et al. 2019). The corresponding constraints on the richness-mass relation when $B_{z}=0$ are also consistent with that of Bleem et al. (2020) shown in Fig. 6. 
Table 3. A comparison of cosmological analyses as summarised in Fig. 7. Columns include references, the origin of the data source, the total number of clusters used in the fiducial analysis, the area covered in square degrees. The final column correspond to the redshift range, i.e. the minimum and maximum cluster redshift used in the sample (analyses are not necessarily complete over this redshift range). The description in parentheses also specifies the type of redshifts used; spectroscopic (spec), photometric (photo) or a mixture (both).

\begin{tabular}{|c|c|c|c|c|c|}
\hline Reference & Origin & Selection & \# Clusters & Area $\left[\operatorname{deg}^{2}\right]$ & Redshift (Type) \\
\hline This work & SPIDERS DR16 & X-ray & 691 & 5,350 & $0.10-0.60\left(\mathrm{spec}^{8}\right)$ \\
\hline Pillepich et al. (2018) & eRASS8 forecast (pessimistic) & X-ray & 88,900 & 41,253 & 0.01-2.00 (photo) \\
\hline Zubeldia \& Challinor (2019) & Planck MMF3 + CMB lensing & SZ & 433 & 26,814 & $0.00-1.00$ (both) \\
\hline Bocquet et al. (2019) & $\mathrm{SPT}+$ Chandra + lensing & $\mathrm{SZ}$ & 377 & 2,500 & $0.29-1.13$ (photo) \\
\hline Abbott et al. (2020a) & DES-redMaPPer + lensing & Optical & 6,504 & 1,500 & $0.20-0.65$ (photo) \\
\hline Kirby et al. (2019) & SDSS-redMaPPer + lensing + Chandra & Optical & 6,964 & 9,000 & $0.10-0.30$ (photo) \\
\hline Zu et al. (2014) & MaxBCG + lensing & Optical & 10,815 & 7,398 & $0.10-0.30$ (photo) \\
\hline Mantz et al. (2015) & Chandra archive & X-ray & 224 & - & $0.08-1.06(\mathrm{spec})$ \\
\hline Pacaud et al. (2018) & $X M M-\mathrm{XXL}$ & X-ray & 178 & 50 & $0.05-1.00(\mathrm{spec})$ \\
\hline
\end{tabular}
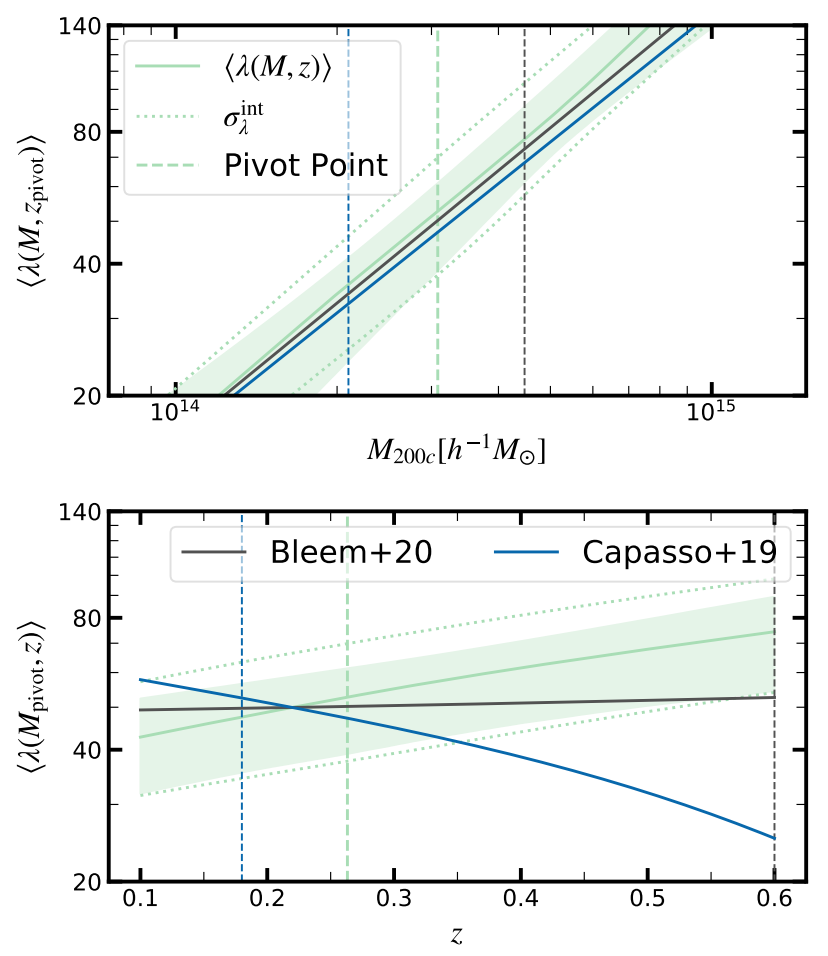

Figure 6. Constraints for the richness-mass relation evaluated at the pivot redshift and mass used in this analysis $(0.263$ and $\left.3.08 \times 10^{14} \mathrm{M}_{\odot} h^{-1}\right)$. The dotted line about the solid line indicates the intrinsic scatter $\left(\sigma_{\text {int }}\right.$ with normal prior from Bleem et al. 2020) about the mean (Eq. 2), while the coloured contours show the 16 and 85 percentiles of the draws from the MCMC chains used to constrain the cosmological and scaling relation parameters shown in Fig. 5.

\subsection{Cosmological constraints}

Table. 3 contains a comparison of cluster count experiments from the literature, with Fig. 7 depicting the respective posterior distributions in the $\Omega_{m_{0}}-\sigma_{8}$ plane. Each experiment is unique in the sense they differ with the primary method of cluster selection and mass calibration, cluster sample size, survey volume and the type of redshifts used in the analysis (photometric, spectroscopic or a mixture of the two):

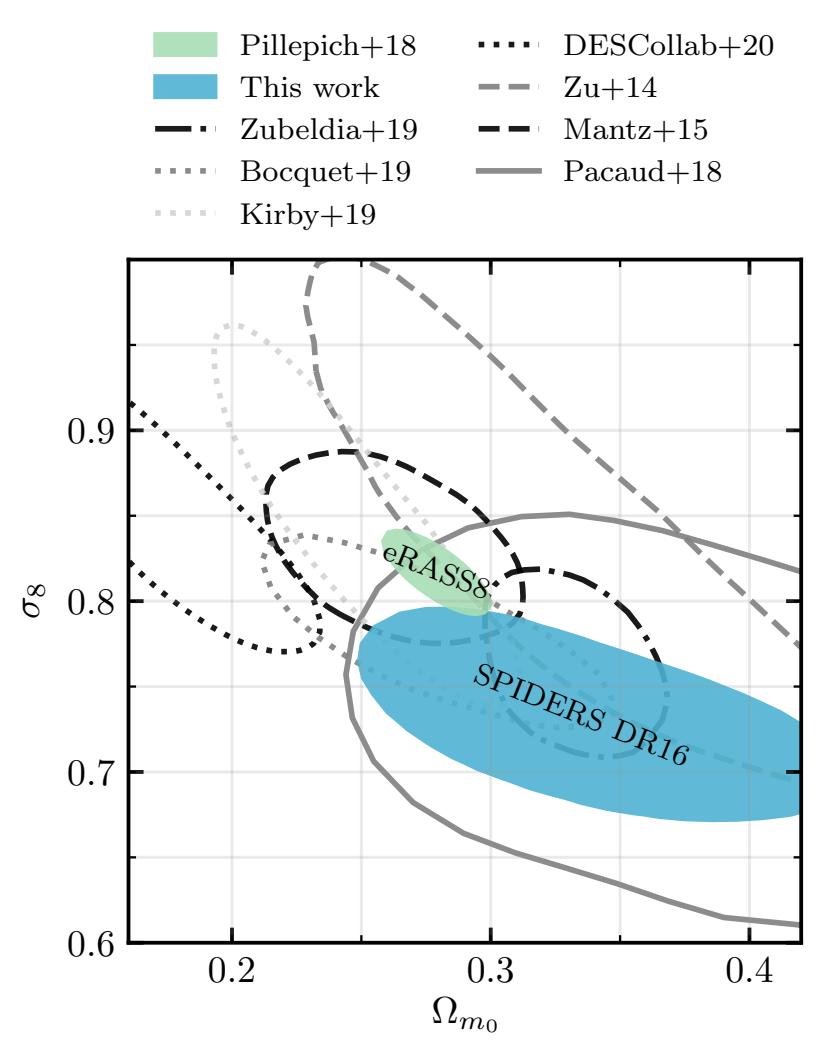

Figure 7. Cosmological parameter constraints obtained with the SPIDERS DR16 compared to previous work. Contours represent the $68 \%$ confidence level. Table. 3 contains a comparison of each reference shown in the figure above, summarising each selection method, sky area, redshift range, sample size, and data origin. The blue ellipse shows the constraints from this work and is identical to the inner contour shown in top-left panel of Fig. 5 when an normal prior is used for the intrinsic scatter of the richnessmass relation (Bleem et al. 2020). The green ellipse represents a conservative forecast of the constraining power of eROSITA after its four year all sky survey (Pillepich et al. 2018). Grey and black lines represent constraints from recent competitive cluster analyses, for more detailed descriptions of each experiment please refer to Sect. 4.2 . 
- Mantz et al. (2015): 224 bright clusters originating from three X-ray flux-limited samples of clusters (Ebeling et al. 1998; Böhringer et al. 2004; Ebeling et al. 2010) based on the RASS (Truemper 1993). Mass proxies are provided from Xray follow-up (94 clusters have high-quality Chandra data), weak gravitational lensing data (50 massive clusters) and measurements of the X-ray gas fraction.

- Pacaud et al. (2018): a sub-sample of 178 bright and spectroscopically confirmed serendipitous clusters from the $X M M$-XXL survey (Adami et al. 2018) with an X-ray selection function depending on the extent and extentlikelihood of the emission to ensure contamination levels are $\lesssim 50 \%$ (Pacaud et al. 2006; Clerc et al. 2012; Pacaud et al. 2016). The redshift range is large, $\tilde{z} \in(0.05,1.00)$, and masses are estimated using calibrated relations between mass-temperature (Lieu et al. 2016), luminositytemperature (Adami et al. 2018) and the link between extent and core-radius (Pacaud et al. 2018).

- Pillepich et al. (2018): a forecast of cosmological constraints predicted from eight scans of eROSITA all sky survey (eRASS8). This version of the forecast is a conservative (pessimistic) estimate of the precision in the sense that only photometric redshifts are used.

- Zubeldia \& Challinor (2019): 439 clusters from the MMF3 cosmology sample (Planck Collaboration et al. 2016c) with a thermal SZ signal-to-noise ratio greater than 6. CMB lensing mass estimates are provided for of all clusters with redshifts (Planck Collaboration et al. 2016a) which originate from a mixture a variety of ancillary data (Planck Collaboration et al. 2014, 2015a,b, 2016b,c). This includes a mixture of photometric and spectroscopic information from X-ray (Piffaretti et al. 2011), optical (Rykoff et al. 2014; Liu et al. 2015) and SZ (Hasselfield et al. 2013; Bleem et al. 2015) counterparts.

- Bocquet et al. (2019): 377 clusters from the South Pole Telescope 2500deg ${ }^{2}$ survey (de Haan et al. 2016) with high detection significance $(\zeta>5)$ a purity of $95 \%$ (Bleem et al. 2015 ). The analysis uses a mixture of photometric (Bleem et al. 2015; Strazzullo et al. 2019) and spectroscopic (Bayliss et al. 2016; Khullar et al. 2019) redshifts over $\tilde{z} \in(0.29,1.13)$ with complementary Chandra X-ray data (McDonald et al. 2013, 2017) for 89 clusters and weak-lensing data from the Hubble Space Telescope and Magellan (Schrabback et al. 2018; Dietrich et al. 2019) for 32 clusters.

The following optical selected cluster analyses all simultaneously model the weak lensing measurement, richnessmass relation and abundance of clusters using photometric redshifts:

- Zu et al. (2014): a sub-sample of 10,815 SDSS MaxBCG clusters (Koester et al. 2007a) which is almost volumelimited over the redshift range $\tilde{z} \in[0.1,0.3)$ with $\sim 90 \%$ completeness and purity for $M_{200 m}>=10^{14} h^{-1} \mathrm{M}_{\odot}$.

- Kirby et al. (2019): improve on the work of Costanzi et al. (2019b), which is based on 6,964 SDSS redMaPPer clusters, by deriving X-ray gas masses for the 30 richest systems using supplementary Chandra data. The cluster sample is volume limited with $\tilde{z} \in[0.1,0.3]$.

- Abbott et al. (2020a): a volume-limited sample of 6,504 DES redMaPPer clusters over $\tilde{z} \in[0.2,0.65]$. Changes to the original Costanzi et al. (2019b) model were implemented after un-blinding, including an updated (tighter) prior on the intrinsic scatter and corrections to account for systematic biases in the weak lensing mass estimates. The contours shown in Fig. 5 correspond to the results of the un-blinded analysis.

The work presented here is differs from all of the above in that it is the only volume-limited X-ray selected cluster sample that uses a self calibrated richness-mass relation as well spectroscopic redshifts over a large area and redshift range without supplementary mass measurements. The Xray selection function does, however, assume a pre-calibrated $\mathrm{X}$-ray luminosity-mass relation, with the magnitude of the shift between the Mantz et al. (2015) constraints and the SPIDERS DR16 constraints expected to represent the order of systematic uncertainty caused by fixing this relation. As the accuracy of the mass-calibration process is usually the largest source of systematic uncertainty impacting cosmological analyses with clusters, the difference between constraints presented in this work and that of SZ selected samples (Zubeldia \& Challinor 2019; Bocquet et al. 2020) is expected to be driven primarily by the ability to accurately constrain observable-mass relation parameters, especially at high-mass and high-redshift. The deviation from the constraints of Kirby et al. (2019) and Abbott et al. (2020a) is partially explained by a more sophisticated physically motivated model of richness related biases compared to this work (Sect. 5.1.3), but mostly due to the systematic effects that impact the weak-lensing mass measurements of optically selected redMaPPer clusters. Although in general, the constraints derived from the SPIDERS DR16 cosmological cluster sub-sample are in good agreement with previously published measurements, the systematic uncertainties caused the X-ray selection function alone are estimated to be least $\gtrsim 20 \%$ (Sect. 5.1.4), with an additional unknown contribution from richness-related biases (Sect. 5.1.3) which have yet to be quantified for CODEX clusters.

\subsection{Impact of intrinsic scatter}

When the intrinsic scatter $\left(\sigma_{\text {int }}\right)$ of the richness-mass relation is assumed to be independent of mass and redshift (Eq. 3), it has shown to be degenerate with the normalisation $(A)$ of the relation (e.g Bleem et al. 2020) as well as with cosmological parameters (Costanzi et al. 2019b). In the case of richness-mass relations derived from a sub-sample of CODEX clusters; $\sigma_{\text {int }}$ and $A$ are consistently found to be almost completely degenerate (e.g. Capasso et al. 2019, Kiiveri et al. subm.). This strong degeneracy between $\sigma_{\text {int }}$ and $A$ is also apparent in this work (Fig. 5), with $\sigma_{\text {int }}$ being a prior dominated quantity. When a restrictive prior is used $\sigma_{\text {int }} \in[0.1,0.3)$, the degeneracy is less obvious, but using the wider uniform prior used by Abbott et al. (2020a) $\sigma_{\text {int }} \in[0.1,0.5)$, the degeneracy is much more significant. This is also the case when using normal priors centred on the best fit values from recently calibrated richness-mass relations (Saro et al. 2015; Capasso et al. 2019; Mulroy et al. 2019; Bleem et al. 2020, and Kiiveri et al. subm.) e.g. $\sigma_{\text {int }} \approx \mathcal{N}(0.2,0.15)$. This choice of prior also effects a mild bimodality in the posterior distribution of the intrinsic scatter. This is likely related to the wide prior used for the normalisation, the poorly constrained redshift evolution parameter and large residuals between the data and model at the most extreme values of richness and redshift shown in Fig. 2. As 
the intrinsic scatter is poorly constrained, this in turn, directly impacts the posterior distribution of the normalisation and mass evolution, although cosmological parameters and the redshift trend of the richness-mass relation are relatively unaffected. This effect, although less prominent, is also observed in the posterior distributions of simulated SPIDERS DR16 catalogue (Fig. B4). Although informative, using a Gaussian prior for the intrinsic scatter centred on the best fit value of Bleem et al. (2020) allows for the widest possible solutions cosmological analysis of the observational dataset (Table. 2) and simulated catalogue (Table. 2). For this reason, best fit values of all parameters are consistently stated for runs which adopt the Bleem et al. (2020) prior.

\section{DISCUSSION}

The input cosmological and richness-mass relation parameters are successfully retrieved using the likelihood model on the simulated catalogue in the validation procedure (Appendix B). This confirms that there are no major numerical issues with the cosmological pipeline. However, as the observed data (Fig. 2) appears much noisier than the simulated data (Fig. B2) it is apparent that it is considerably more complex than the model employed in this work. Failure to account for such complexities can cause shifts in the measured parameters relative to their true value. This section discusses several possible sources of systematic uncertainties and their relevance in the context of this work.

\subsection{Systematic uncertainties}

\subsubsection{Mass function systematics}

The systematic uncertainty imposed by the chosen HMF model, is subdominant relative to the measured precision for all constrained parameters. This is confirmed by recovering almost identical posterior distributions after repeating the abundance modelling with and a Tinker et al. (2008), Bocquet et al. (2016) and Despali et al. (2016) multiplicity function in Eq. 10. In theory, this source of uncertainty could be reduced further by using a high accuracy HMF emulator (e.g. McClintock et al. 2019b; Nishimichi et al. 2019; Bocquet et al. 2020). However, as this analysis assumes $h=0.7$, the cosmological parameter space covered by the majority of the (publicly available) emulators is not sufficiently wide to cover the broad posterior distributions of $\Omega_{m_{0}}$ and $\sigma_{8}$.

\subsubsection{Redshift bias}

The benefit of using spectroscopic redshifts means that the redshifts are extremely accurate (unbiased) relative to photometric redshifts (Clerc et al. 2020, Kirkpatrick et al. subm.), therefore there is no contribution to the systematic uncertainty due to photometric redshift estimation. The median statistical uncertainties of spectroscopic redshifts (Sect. 2.3) within the SPIDERS DR16 sample over the redshift range considered in this analysis, $z \in[0.1,0.6)$, is of the order $\Delta_{z} /(1+\tilde{z}) \sim 7 \times 10^{-4}$. When compared to photometric redshifts derived from the SDSS (the DESI Legacy Imaging Surveys; Sect. 2.2), equivalent statistical uncertainties are found to be $\sim 0.0097(0.0075)$, meaning the impact of redshift measurement uncertainties is reduced by a factor of 13 (10). SPIDERS DR16 spectroscopic redshifts are therefore an excellent approximation to the true cluster redshift, allowing one to safely avoid marginalising over the redshift uncertainties when modelling of the abundance of SPIDERS clusters (i.e. the PDF which considers the uncertainties on observed redshifts in Eq. 8 can be approximated as a Dirac Delta function).

\subsubsection{Richness bias}

During the visual inspection procedure within the SPIDERS cluster pipeline (Sect. 2.3), it is possible to disentangle structure along the line of site. This reduces the impact that projection effects have on the number of redshift outliers, with $\sim 10 \%$ of SPIDERS systems being multi-component (Clerc et al. 2020). The value of richness, however, can still be severely affected, usually resulting in up-scattered richness values for structure which cannot be de-blended. Ignoring this effect has been shown to systematically shift $\sigma_{8}$ high and $\Omega_{m_{0}}$ low, relative to their true values and can increase the instrinsic scatter (Costanzi et al. 2019a). Recent cosmological analyses using optically selected clusters (Costanzi et al. 2019b; Abbott et al. 2020a) include specially designed terms within their abundance model to account for biases caused by projection effects. For SPIDERS, however, an equivalent term has yet to be derived. To study this effect in detail requires accurate methods to jointly mock X-ray observables and optical richness, which will be addressed in future work.

Percolation effects describe the process of assigning member galaxies which appear in multiple cluster candidates to the cluster with the maximum redMaPPer likelihood (Rykoff et al. 2014). In some cases, this can cause low-richness systems to be blended into richer systems when aligned along the line of sight. This results in a deficiency in the number of low-richness systems and higher values of richness for systems which are assigned the additional member galaxies. Modifying the percolation radii parameters to extreme values when constructing the DES redMaPPer cluster sample was shown to have a cosmologically negligible impact on the total number of systems (Abbott et al. 2020a). Although, the impact is also expected to be small for this study, percolation effects have yet to be quantified for the CODEX cluster sample when measuring richness with redMaPPer configured in scanning-mode.

The optical centre chosen by redMaPPer also has a systematic effect on the value of observed richness as it defines the radial aperture over which the membership probabilities of galaxies are summed. The performance of centring algorithm(s) in redMaPPer has been studied at length (e.g. Hoshino et al. 2015; Hikage et al. 2018) with a focus on comparisons to fiducial X-ray selected centres from high-quality archival data (Rozo \& Rykoff 2014; Zhang et al. 2019). As Xrays trace the dominant component of baryonic mass in the ICM, X-ray centres are in general more closely linked to the true centre for relaxed clusters. However, as CODEX clusters are detected using ROSAT data, the positional accuracy of the X-ray centres is $\sim 3$ arcmin (Clerc et al. 2012). This means that on average, optical centres found by redMaPPer, which use prior information of CODEX X-ray source positions, are expected to be much closer to the fiducial cluster centres than the priors themselves. 
For SPIDERS clusters analysed with The Legacy Surveys with $\tilde{\lambda}>20$, the mean distance between optical centre determined by redMaPPer and the original RASS Xray source is found to be $0.19 \mathrm{Mpc}$ with a standard deviation of $0.10 \mathrm{Mpc}$, however, whether or not the optical centres of CODEX clusters analysed with The Legacy Surveys are more accurate than those found in purely optical selected clusters has yet to be studied in detail. Given that the difference between the value of richness at the optical centre and the mean value of richness averaged over the five most likely centres (within of $0.40 \mathrm{Mpc}$ of the X-ray centre) is less than the measured uncertainty on the richness for $99.4 \%$ of systems, it is unlikely that centring issues significantly contribute to the systematic uncertainties of this analysis.

\subsubsection{Selection bias}

Selection bias is the largest contribution to the total error budget. This is because the parameters of the X-ray luminosity-mass relation used in the construction of the CODEX selection function are fixed (Lieu et al. 2016; Giles et al. 2016) along with $h=0.7$ and $\Omega_{m_{0}}=0.30$ (Finoguenov et al. 2020). This causes any inaccuracies in these parameters to be absorbed into the posterior distributions of the richness-mass relation and cosmological parameters. In an attempt to minimise the overall systematic impact of these fixed parameters, only systems which are above the 50\% sensitivity limit of the survey are taken into consideration when defining the cosmological cluster sub-sample (Sect. 3.2.3). Assuming these parameters of the X-ray luminosity-mass are known within $10 \%$ of their true values, the resulting contribution to the systematic uncertainty on the abundance prediction would be a maximum of $\sim 20 \%$. Conducting the cosmological analysis without marginalising over $\Omega_{m_{0}}$ in the $\mathrm{X}$-ray selection function, however, is estimated ${ }^{9}$ to cause systematic uncertainties on the richness-mass relation of at least $5 \%$ on the normalisation, $8 \%$ on the mass evolution and $50 \%$ on the redshift evolution. The respective impact on cosmological parameters is $\sim 20 \%$ for $\Omega_{m_{0}}$ and $\sim 15 \%$ for $\sigma_{8}$.

\section{SUMMARY}

$\Omega_{m}$ and $\sigma_{8}$ are constrained along with the parameters of the richness-mass scaling relation using a high-purity sub-sample of 691 CODEX clusters over the SPIDERS DR16 footprint $\left(5,350 \mathrm{deg}^{2}\right)$ after a reanalysis of the original CODEX source catalogue using redMaPPer and The Legacy Surveys. The cluster abundance is modelled by considering the X-ray selection, a redshift dependant cut in observedrichness and the selection effect due to applying redMaPPer to The Legacy Surveys in bins of observed richness and redshift. The value of having spectroscopic redshifts is demonstrated via a fine-grain binning strategy that enables a relatively high cosmological constraining power. Despite

\footnotetext{
9 Systematic uncertainties are estimated by repeating the cosmological analysis on the data with $\Omega_{m_{0}}=0.24$ and $\Omega_{m_{0}}=0.36$ fixed in the X-ray selection function, $P\left(I_{\mathrm{X}} \mid \mu, z, v\right)$, with a restrictive prior on the intrinsic scatter $\sigma_{\text {int }} \in[0.1,0.3]$.
}

this, the extrapolation of a complex selection model in the high redshift regime leads to underestimated statistical uncertainties due to its fixed cosmology and luminosity-mass relation. This also contributes to the systematics that affect the redshift evolution of the richness-mass relation which indicates an increasing over-abundance of rich clusters with redshift given the best-fit cosmology. In order to improve the results presented here, it is necessary to marginalise over all cosmologically dependent parameters including the Hubble constant, baryon fraction, neutrinos etc. as well as the luminosity-mass and richness-mass scaling relations in all aspects of the model (e.g. selection processes and HMF emulation). To improve the accuracy of the model, the likelihood function should also be modified to include projection/percolation effects and consider sample variance in the covariance matrix.

Modelling the low-photon statistics of RASS combined with optical richness from The Legacy Surveys is challenging, and the quality of the X-ray and photometric data can be improved to facilitate the usage of lower-scatter mass proxies which also simplify the selection modelling. The selfcalibration procedure of the richness-mass relation would also largely benefit from accurate mass measurements from gravitational lensing, dynamics and/or high quality X-ray follow up (e.g. Mantz et al. 2015; Kirby et al. 2019; Bocquet et al. 2020). The combination of eROSITA (Merloni et al. 2012), wide/deep photometric surveys such as LSST (Ivezić et al. 2019) and multi-object spectrograph like 4MOST (de Jong et al. 2012) and DESI (DESI Collaboration et al. 2016) will provide the next generation of large spectroscopic cluster samples. These will benefit from the investments into the SPIDERS programme and supersede the DR16 dataset to provide high-precision competitive cosmological constraints with well understood systematics.

\section{DATA AVAILABILITY}

The cosmological sub-sample of CODEX clusters reanalysed with The Legacy Surveys is publicly available at https://www.mpe.mpg.de/XraySurveys/SPIDERS. This catalogue includes X-ray and optical centres, spectroscopic and photometric redshifts, richness, centring probabilities as well as a catalogue of member galaxies. Additionally, the cosmological likelihood code, the custom version of redMaPPer as well as the optical and spectroscopic processing pipelines can be found at https://github.com/jacobic/spiders-cosmology.git. For any additional data or code requests please contact jacobic@mpe.mpg.de.

\section{REFERENCES}

Abazajian K., Dodelson S., 2003, Phys. Rev. Lett., 91, 041301 Abbott T. M. C., et al., 2020a, Phys. Rev. D, 102, 023509

Abbott T. M. C., et al., 2020b, Phys. Rev. D, 102, 023509

Adami C., et al., 2018, A\&A, 620, A5

Ahumada R., et al., 2020, ApJS, 249, 3

Aihara H., et al., 2011, The Astrophysical Journal Supplement Series, 193, 29

Alam S., et al., 2017, MNRAS, 470, 2617

Allen S. W., Evrard A. E., Mantz A. B., 2011, ARA\&A, 49, 409 
Astropy Collaboration et al., 2013, A\&A, 558, A33

Astropy Collaboration et al., 2018, AJ, 156, 123

Ata M., et al., 2018, MNRAS, 473, 4773

Bahcall N. A., Cen R., 1993, ApJ, 407, L49

Bahcall N. A., et al., 2003, ApJ, 585, 182

Bardeen J. M., Bond J. R., Kaiser N., Szalay A. S., 1986, ApJ, 304, 15

Bayliss M. B., et al., 2016, ApJS, 227, 3

Becker M. R., et al., 2007, ApJ, 669, 905

Beutler F., et al., 2011, MNRAS, 416, 3017

Birkinshaw M., 1999, Phys. Rep., 310, 97

Blanton M. R., et al., 2017, AJ, 154, 28

Bleem L. E., et al., 2015, ApJS, 216, 27

Bleem L. E., et al., 2020, ApJS, 247, 25

Bocquet S., Saro A., Dolag K., Mohr J. J., 2016, MNRAS, 456, 2361

Bocquet S., et al., 2019, ApJ, 878, 55

Bocquet S., Heitmann K., Habib S., Lawrence E., Uram T., Frontiere N., Pope A., Finkel H., 2020, ApJ, 901, 5

Böhringer H., et al., 2000, ApJS, 129, 435

Böhringer H., et al., 2004, A\&A, 425, 367

Böhringer H., Chon G., Collins C. A., 2014, A\&A, 570, A31

Borgani S., Guzzo L., 2001, Nature, 409, 39

Bruzual G., Charlot S., 2003, MNRAS, 344, 1000

Burke C., Hilton M., Collins C., 2015, MNRAS, 449, 2353

Capasso R., et al., 2019, MNRAS, 486, 1594

Child H. L., Habib S., Heitmann K., Frontiere N., Finkel H., Pope A., Morozov V., 2018, ApJ, 859, 55

Clerc N., Sadibekova T., Pierre M., Pacaud F., Le Fèvre J.-P., Adami C., Altieri B., Valtchanov I., 2012, MNRAS, 423, 3561

Clerc N., et al., 2016, MNRAS, 463, 4490

Clerc N., et al., 2020, MNRAS,

Comparat J., et al., 2020, arXiv e-prints, p. arXiv:2008.08404

Conroy C., Wechsler R. H., Kravtsov A. V., 2007, ApJ, 668, 826

Costanzi M., et al., 2019a, MNRAS, 482, 490

Costanzi M., et al., 2019b, MNRAS, 488, 4779

DESI Collaboration et al., 2016, preprint, (arXiv:1611.00036)

Dalcín L., Paz R., Storti M., 2005, Journal of Parallel and Distributed Computing, 65, 1108

Dalcín L., Paz R., Storti M., D’Elía J., 2008, Journal of Parallel and Distributed Computing, 68, 655

Dalcin L. D., Paz R. R., Kler P. A., Cosimo A., 2011, Advances in Water Resources, 34, 1124

Dawson K. S., et al., 2016, AJ, 151, 44

Despali G., Giocoli C., Angulo R. E., Tormen G., Sheth R. K., Baso G., Moscardini L., 2016, MNRAS, 456, 2486

Dey A., et al., 2019, AJ, 157, 168

Diemer B., 2018, ApJS, 239, 35

Diemer B., Kravtsov A. V., 2015, ApJ, 799, 108

Dietrich J. P., et al., 2019, MNRAS, 483, 2871

Ebeling H., Edge A. C., Bohringer H., Allen S. W., Crawford C. S., Fabian A. C., Voges W., Huchra J. P., 1998, MNRAS, 301,881

Ebeling H., Edge A. C., Mantz A., Barrett E., Henry J. P., Ma C. J., van Speybroeck L., 2010, MNRAS, 407, 83

Eddington A. S., 1913, MNRAS, 73, 359

Eisenstein D. J., et al., 2005, ApJ, 633, 560

Finoguenov A., et al., 2020, A\&A, 638, A114

Fixsen D. J., 2009, ApJ, 707, 916

Foreman-Mackey D., Hogg D. W., Lang D., Goodman J., 2013, PASP, 125, 306

Foreman-Mackey D., et al., 2020, dfm/corner.py: corner.py v2.1.0.rc1, doi:10.5281/zenodo.3937526, https://doi.org/ 10.5281/zenodo. 3937526

Fukugita M., Ichikawa T., Gunn J. E., Doi M., Shimasaku K., Schneider D. P., 1996, AJ, 111, 1748

Giles P. A., et al., 2016, A\&A, 592, A3

Gladders M. D., Yee H. K. C., 2005, ApJS, 157, 1
Gladders M. D., Yee H. K. C., Majumdar S., Barrientos L. F., Hoekstra H., Hall P. B., Infante L., 2007, ApJ, 655, 128

Górski K. M., Hivon E., Banday A. J., Wandelt B. D., Hansen F. K., Reinecke M., Bartelmann M., 2005, ApJ, 622, 759

Grandis S., et al., 2020, arXiv e-prints, p. arXiv:2002.10834

Gunn J. E., et al., 2006, AJ, 131, 2332

Hasselfield M., et al., 2013, J. Cosmology Astropart. Phys., 2013, 008

Hikage C., Mandelbaum R., Leauthaud A., Rozo E., Rykoff E. S., 2018, MNRAS, 480, 2689

Hogg D. W., 1999, ArXiv Astrophysics e-prints,

Hoshino H., et al., 2015, MNRAS, 452, 998

Hu W., Kravtsov A. V., 2003, ApJ, 584, 702

Hunter J. D., 2007, Computing In Science \& Engineering, 9, 90

Ivezić Ž., et al., 2019, ApJ, 873, 111

Jones D. O., et al., 2018, ApJ, 857, 51

Jullo E., et al., 2019, A\&A, 627, A137

Kellogg E., Baldwin J. R., Koch D., 1975, ApJ, 199, 299

Khullar G., et al., 2019, ApJ, 870, 7

Kirby M., Rozo E., Morris R. G., Allen S. W., Costanzi M., Mantz A. B., Rykoff E. S., von der Linden A., 2019, arXiv e-prints, p. arXiv:1910.13548

Klein M., et al., 2018, MNRAS, 474, 3324

Klein M., et al., 2019, MNRAS, 488, 739

Klypin A., Yepes G., Gottlöber S., Prada F., Heß S., 2016, MNRAS, 457, 4340

Koester B. P., et al., 2007a, ApJ, 660, 221

Koester B. P., et al., 2007b, ApJ, 660, 239

Lieu M., et al., 2016, A\&A, 592, A4

Liu J., et al., 2015, MNRAS, 449, 3370

Mana A., Giannantonio T., Weller J., Hoyle B., Hütsi G., Sartoris B., 2013, MNRAS, 434, 684

Mantz A. B., et al., 2015, MNRAS, 446, 2205

McClintock T., et al., 2019a, MNRAS, 482, 1352

McClintock T., et al., 2019b, ApJ, 872, 53

McDonald M., et al., 2013, ApJ, 774, 23

McDonald M., et al., 2017, ApJ, 843, 28

Merloni A., et al., 2012, preprint, (arXiv:1209.3114)

Mulroy S. L., et al., 2019, MNRAS, 484, 60

Murata R., et al., 2019, PASJ, 71, 107

Navarro J. F., Frenk C. S., White S. D. M., 1997, ApJ, 490, 493

Nishimichi T., et al., 2019, ApJ, 884, 29

Old L., et al., 2018, MNRAS, 475, 853

Oliphant T. E., 2006, A guide to NumPy. Vol. 1, Trelgol Publishing USA

Pacaud F., et al., 2006, MNRAS, 372, 578

Pacaud F., et al., 2016, A\&A, 592, A2

Pacaud F., et al., 2018, A\&A, 620, A10

Peebles P. J. E., 1980, The large-scale structure of the universe. Princeton University Press

Peloton J., Arnault C., Plaszczynski S., 2018, preprint, (arXiv: 1804.07501)

Perlmutter S., et al., 1999, ApJ, 517, 565

Pezzotta A., et al., 2017, A\&A, 604, A33

Phriksee A., Jullo E., Limousin M., Shan H., Finoguenov A., Komonjinda S., Wannawichian S., Sawangwit U., 2020, MNRAS, 491, 1643

Piffaretti R., Arnaud M., Pratt G. W., Pointecouteau E., Melin J. B., 2011, A\&A, 534, A109

Pillepich A., Reiprich T. H., Porciani C., Borm K., Merloni A., 2018, MNRAS, 481, 613

Planck Collaboration et al., 2014, A\&A, 571, A29

Planck Collaboration et al., 2015a, A\&A, 581, A14

Planck Collaboration et al., 2015b, A\&A, 582, A29

Planck Collaboration et al., 2016a, A\&A, 594, A24

Planck Collaboration et al., 2016b, A\&A, 594, A26

Planck Collaboration et al., 2016c, A\&A, 594, A27

Planck Collaboration et al., 2018, arXiv e-prints, 
Planck Collaboration et al., 2020, A\&A, 641, A6

Plaszczynski S., Peloton J., Arnault C., Campagne J. E., 2019, Astronomy and Computing, 28, 100305

Pratt G. W., Arnaud M., Biviano A., Eckert D., Ettori S., Nagai D., Okabe N., Reiprich T. H., 2019, Space Sci. Rev., 215, 25

Reiprich T. H., Böhringer H., 2002, ApJ, 567, 716

Riess A. G., et al., 1998, AJ, 116, 1009

Riess A. G., et al., 2018a, ApJ, 853, 126

Riess A. G., et al., 2018b, ApJ, 861, 126

Ross A. J., Samushia L., Howlett C., Percival W. J., Burden A., Manera M., 2015, MNRAS, 449, 835

Rozo E., Rykoff E. S., 2014, ApJ, 783, 80

Rozo E., et al., 2010, ApJ, 708, 645

Rykoff E. S., et al., 2012, ApJ, 746, 178

Rykoff E. S., et al., 2014, ApJ, 785, 104

Rykoff E. S., Rozo E., Keisler R., 2015, preprint, (arXiv: 1509.00870)

Rykoff E. S., et al., 2016, ApJS, 224, 1

Sadibekova T., Pierre M., Clerc N., Faccioli L., Gastaud R., Le Fevre J.-P., Rozo E., Rykoff E., 2014, A\&A, 571, A87

Saro A., et al., 2015, MNRAS, 454, 2305

Schrabback T., et al., 2018, MNRAS, 474, 2635

Scolnic D. M., et al., 2018, ApJ, 859, 101

Sheth R. K., Tormen G., 2002, MNRAS, 329, 61

Smee S. A., et al., 2013, AJ, 146, 32

Strazzullo V., et al., 2019, A\&A, 622, A117

Sunyaev R. A., Zeldovich Y. B., 1970, Ap\&SS, 7, 3

Sunyaev R. A., Zeldovich Y. B., 1972, Comments on Astrophysics and Space Physics, 4, 173

Swanson M. E. C., Tegmark M., Hamilton A. J. S., Hill J. C., 2008, MNRAS, 387, 1391

Szabo T., Pierpaoli E., Dong F., Pipino A., Gunn J., 2011, ApJ, 736,21

Taylor M. B., 2005, in Shopbell P., Britton M., Ebert R., eds, Astronomical Society of the Pacific Conference Series Vol. 347, Astronomical Data Analysis Software and Systems XIV. p. 29

Team T. P. D., 2020, pandas-dev/pandas: Pandas, doi:10.5281/zenodo.3509134, https://doi.org/10.528 1/zenodo. 3509134

The Dark Energy Survey Collaboration 2005, arXiv e-prints, pp astro-ph/0510346

Tinker J., Kravtsov A. V., Klypin A., Abazajian K., Warren M., Yepes G., Gottlöber S., Holz D. E., 2008, ApJ, 688, 709

Tinker J. L., et al., 2012, ApJ, 745, 16

Truemper J., 1993, Science, 260, 1769

Umetsu K., 2020, arXiv e-prints, p. arXiv:2007.00506

Vanderlinde K., et al., 2010, ApJ, 722, 1180

Verde L., Treu T., Riess A. G., 2019, Nature Astronomy, 3, 891

Vikhlinin A., et al., 2009a, ApJ, 692, 1033

Vikhlinin A., et al., 2009b, ApJ, 692, 1060

Virtanen P., et al., 2019, arXiv e-prints, p. arXiv:1907.10121

Voges W., et al., 1999, A\&A, 349, 389

Weinberg D. H., Mortonson M. J., Eisenstein D. J., Hirata C., Riess A. G., Rozo E., 2013, Phys. Rep., 530, 87

Wen Z. L., Han J. L., Liu F. S., 2010, MNRAS, 407, 533

Zarrouk P., et al., 2018, MNRAS, 477, 1639

Zentner A. R., 2007, International Journal of Modern Physics D, 16,763

Zhang Y., et al., 2019, MNRAS, 487, 2578

Zu Y., Weinberg D. H., Rozo E., Sheldon E. S., Tinker J. L., Becker M. R., 2014, MNRAS, 439, 1628

Zubeldia Í., Challinor A., 2019, MNRAS, 489, 401

Zwicky F., 1933, Helvetica Physica Acta, 6, 110

de Haan T., et al., 2016, ApJ, 832, 95

de Jong R. S., et al., 2012, in Ground-based and Airborne Instrumentation for Astronomy IV. p. 84460T (arXiv:1206.6885), doi: $10.1117 / 12.926239$ van der Walt S., Colbert S. C., Varoquaux G., 2011, Computing in Science and Engineering, 13, 22

\section{ACKNOWLEDGEMENTS}

The authors would like to thank the anonymous referee for their patience and useful comments. The first author also thanks Kimmo Kiiveri, Matthias Klein, Joe Mohr, Sebastian Grandis, Mara Salvato, Riccardo Arcodia and Damien Coffey for their scientific advice and personal support during the preparation of this research paper.

This paper represents an effort by both the SDSSIII and SDSS-IV collaborations. Funding for SDSS-III was provided by the Alfred P. Sloan Foundation, the Participating Institutions, the National Science Foundation, and the U.S. Department of Energy Office of Science. Funding for the Sloan Digital Sky Survey IV has been provided by the Alfred P. Sloan Foundation, the U.S. Department of Energy Office of Science, and the Participating Institutions. SDSS-IV acknowledges support and resources from the Center for High-Performance Computing at the University of Utah. The SDSS web site is www.sdss.org. SDSS-IV is managed by the Astrophysical Research Consortium for the Participating Institutions of the SDSS Collaboration including the Brazilian Participation Group, the Carnegie Institution for Science, Carnegie Mellon University, the Chilean Participation Group, the French Participation Group, Harvard-Smithsonian Center for Astrophysics, Instituto de Astrofísica de Canarias, The Johns Hopkins University, Kavli Institute for the Physics and Mathematics of the Universe (IPMU) / University of Tokyo, Lawrence Berkeley National Laboratory, Leibniz Institut für Astrophysik Potsdam (AIP), Max-Planck-Institut für Astronomie (MPIA Heidelberg), Max-Planck-Institut für Astrophysik (MPA Garching), Max-Planck-Institut für Extraterrestrische Physik (MPE), National Astronomical Observatory of China, New Mexico State University, New York University, University of Notre Dame, Observatário Nacional / MCTI, The Ohio State University, Pennsylvania State University, Shanghai Astronomical Observatory, United Kingdom Participation Group, Universidad Nacional Autónoma de México, University of Arizona, University of Colorado Boulder, University of Portsmouth, University of Utah, University of Virginia, University of Washington, University of Wisconsin, Vanderbilt University, and Yale University.

This work makes extensive use the SOA/NASA Astrophysics Data System, TOPCAT astronomical data analysis software (Taylor 2005) and spark-fits (Peloton et al. 2018; Plaszczynski et al. 2019). Data processing is carried out with the Draco and Cobra High Performance Computing Systems, located at the Max Planck Computing and Data Facility. The author acknowledges the following python packages: mpi4py (Dalcín et al. 2005, 2008; Dalcin et al. 2011), astropy (Astropy Collaboration et al. 2013, 2018), numpy (Oliphant 2006; van der Walt et al. 2011), scipy (Virtanen et al. 2019), pandas (Team 2020), matplotlib (Hunter 2007) and corner (Foreman-Mackey et al. 2020). 


\section{APPENDIX A: REDMAPPER CONFIGURATION FOR THE LEGACY SURVEYS}

The re-analysis of the SPIDERS cluster sample using The Legacy Surveys is based on a redMaPPer run from $\tilde{z} \in$ $[0.05,0.72$ ) with a z-band (reference band) magnitude limit of 23.5. This makes use of $g-r$ and $r-z$ colours with a transition between the two at a redshift of 0.35 . The spectroscopic training set used for the calibration of the default Bruzual \& Charlot (2003) red-sequence models easily exceeds the minimum redMaPPer requirements over the redshift range of interest (Rykoff et al. 2014). This calibration procedure is carried out over the entire area of The Legacy Surveys, which uses all available spectroscopic galaxies in the literature, and is repeated for three iterations to ensure convergence.

Although the most significant improvements to the optical richness and contamination fraction are due to the deeper photometry and spectroscopic richness, there are also modest improvements due to updates within the redMaPPer algorithm itself (Rykoff et al. 2016). For instance, the estimation of the colour background is more accurate in redMaPPer v6.6 (relative to v5.2 for CODEX), due to the improved consideration of the local masking information and maps of limiting magnitude that capture the systematic variations in the photometry as a function of sky position. The treatment of depth related systematic effects is vital because the observing strategies and depth of each of contributing imaging survey are largely contrasting.

The redMaPPer depth map is generated via a parametric depth model Rykoff et al. (2015) at a maximum resolution of healpix NSIDE=4096 (Górski et al. 2005). If a healpixel has an an insufficient number of galaxies for the model to converge, the depth is approximated by expanding out to the next largest pixel in the nested scheme until it does. The corresponding mask is generated from the bitmasks set in The Legacy Surveys random point catalogues ${ }^{10}$. Points that reduce the fraction of good coverage from unity have the following bitmasks ${ }^{11}$ bits set: BRIGHT, MEDIUM, SATUR_Z, ALLMASK_Z, BAILOUT, GALAXY Or CLUSTER. When producing the galaxy source catalogue for redMaPPer to ingest, all sources with a stellar PSF morphological model type or the NPRIMARY maskbit is not set are also eliminated removed. This removes stellar contamination and duplicates sources in the overlap regions between bricks.

When determining optical richness in this work, redMaPPer is configured in scanning-mode (Rozo \& Rykoff 2014) i.e. it evaluates the optical properties (e.g. richness, redshift, membership and centring probabilities) of an input cluster catalogue at each point on a grid of redshift to determine an initial estimates before remeasuring them without a quantised redshift grid. This mode uses prior knowledge of the positions of cluster centres, producing a sample that is primarily defined by the original selection method used to construct the input catalogue (e.g. X-ray or SZ Finoguenov et al. 2020; Bleem et al. 2020), rather than searching for overdensities of red galaxies in the conventional cluster-finding mode which produces purely optically selected samples. The

10 http://legacysurvey.org/dr8/files/\#random-catalogs

11 http://legacysurvey.org/dr8/bitmasks specific scanning-mode method used in this analysis determines the maximum likelihood richness (and photometric redshift) before the final percolation procedure, this differs from the scanning-mode used to create the original CODEX catalogue (Finoguenov et al. 2020) which determined the maximum richness and respective redshift. The maximum search radius for central galaxy candidates is $400 \mathrm{kpc}$ relative to the initial X-ray (RASS) position. This parameter is unchanged and consistent with Finoguenov et al. (2020).

\section{APPENDIX B: VALIDATION WITH SIMULATIONS}

To conduct a robust test of the cosmological pipeline, a validation procedure is performed using a mock catalogue of clusters (Comparat et al. 2020). The basis of the mock is a suite of MultiDark simulations (Klypin et al. 2016) generated under a Planck cosmology $\left(H_{0}=67.77 \mathrm{kms}^{-1} \mathrm{Mpc}^{-1}\right.$, $\Omega_{m_{0}}=0.307115, \Omega_{b_{0}}=0.048206, \sigma_{8}=0.8228, n_{s}=0.96$, $w_{0}=-1, N_{\text {eff }}=3.046$ ). The simulation (box volume of $1.0 \mathrm{Gpc}^{3}$ ) is replicated to cover the full sky down to halo masses of $M_{500 \mathrm{c}}=5 \times 10^{13} M_{\odot}$ for $\tilde{z} \in[0.1,0.6)$.

Values of observed richness are generated by assuming a log-normal distribution of true richness with a mean equal to the expectation value from the SPTPol Extended Cluster Survey richness-mass relation (Bleem et al. 2020, derived at a Planck-like fiducial cosmology) with a standard deviation determined by Eq. 3. For simplicity, the relation is left in its original form of, i.e. as function of $M_{500 c}$, with a redshift evolution which scales with the Hubble parameter, $E(z) / E\left(z_{\text {pivot }}\right)$, rather than the $(1+z) /\left(1+z_{\text {pivot }}\right)$ scaling assumed in Eq. 2. The SPIDERS DR16 polygon mask (Swanson et al. 2008) is then applied to ensure the coverage of the simulated catalogue matches the survey footprint. The number of clusters in the catalogue is reduced further using weighted down-sampling. Weights are determined for each halo using the probability provided by the CODEX X-ray selection function $P\left(I_{\mathrm{X}} \mid \mu, z, v\right)$ (Finoguenov et al. 2020, assuming $\Omega_{m_{0}}=0.31$ ), which depends only on the true halo properties of the simulated clusters, and the richness-mass relation to transform the generated richness into $v$ space via Eq. 4).

The redshift-mask (derived from a redMaPPer depth map of the limiting galaxy magnitude and models of $m_{*}$ ) is then applied to produce a volume limited catalogue with $L_{*}<0.2$ and $\tilde{\lambda}>5$. The selection term to account for this, $P\left(I_{\text {Legacy }} \mid \ln \tilde{\lambda}, \tilde{z}\right)$, is estimated using random weighted points a for each bin of observed redshift and richness in the simulated catalogue as detailed in Sect. 3.2.2. Finally, a redshift dependant cut in richnes, $P\left(I_{\text {cut }} \mid \ln \tilde{\lambda}, \tilde{z}\right)^{12}$ is applied to the simulated cluster sample to create a synthetic catalogue that resembles the high-purity SPIDERS DR16 sub-sample (Sect. 2.4 \& Eq. 5).

The SPIDERS DR16 likelihood function (Eq. 11) is applied to the synthetic sub-sample to attempt recover the input cosmological and scaling relation parameters. The cos-

12 Please note this is the same redshift dependent redshift cut as for the analysis using the observed CODEX cluster sample (Eq. 5), however, it is kept in terms of the DES definition of richness. 


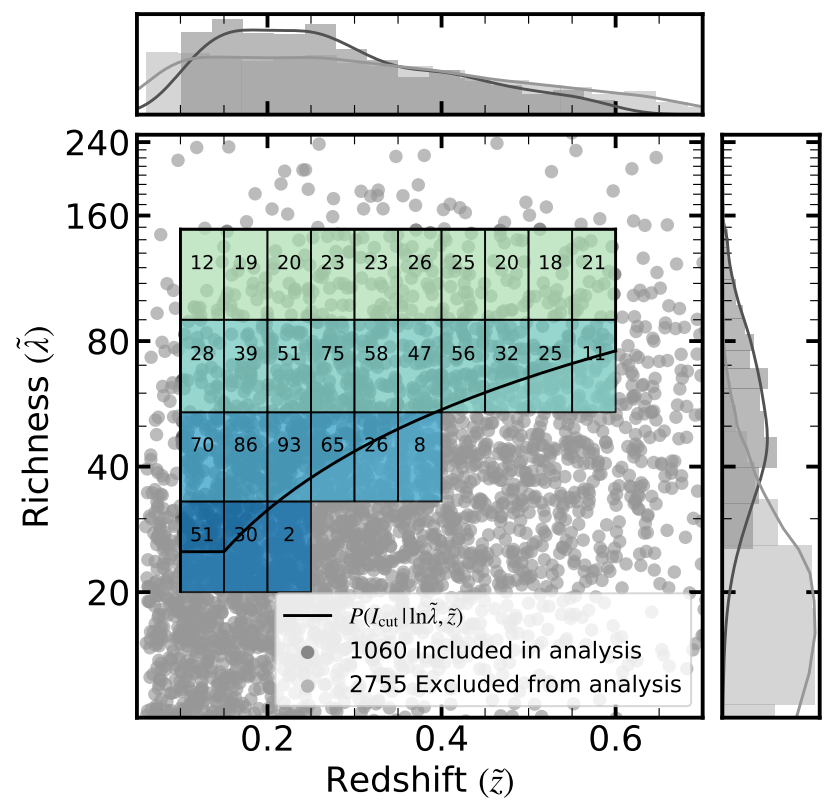

Figure B1. The simulated validation sample of SPIDERS DR16 clusters (3815 in total) in the richness-redshift plane. This is after applying the SPIDERS DR16 survey mask, volume-limiting procedure $\left(L_{*}<0.2\right.$, maximum mask fraction $>0.2$ and $\left.\lambda>5\right)$ and down-sampling using the CODEX X-ray selection function, $P\left(I_{\mathrm{X}} \mid \mu, z, v\right)$. The solid black line represents a redshift-dependant richness cut described by $P\left(I_{\text {cut }} \mid \ln \tilde{\lambda}, \tilde{z}\right)$ in Eq. 5 . Boxes represent the bins used in the validation experiment annotated with the respective clusters counts (after applying $\left.P\left(I_{\text {cut }} \mid \ln \tilde{\lambda}, \tilde{z}\right)\right)$ and colours represent the richness bins highlighted in Fig. B2. The dark grey distributions illustrate clusters that are included in the analysis i.e. clusters that fall within the coloured boxes and that are above the black line. The light grey distributions illustrate clusters excluded from the analysis.

mological parameters are recoverable within one standard deviation of their input values using a Tinker et al. 2008 HMF, see Figs. B2 and B4. In order to establish how accurately the input relation is recovered, the $M_{500 \mathrm{c}}$ definition of the input relation is converted to a function of $M_{200 \mathrm{c}}$ using a Child et al. (2018) halo concentration model and NFW profile (Navarro et al. 1997). The comparison between the measured richness-mass relation and the input relation at the pivot (median) mass and redshift of the simulated dataset is shown in Fig. B3 using consistent definitions of mass. The recovered normalisation, mass and redshift slopes are in good agreement with the input richness-mass relation.

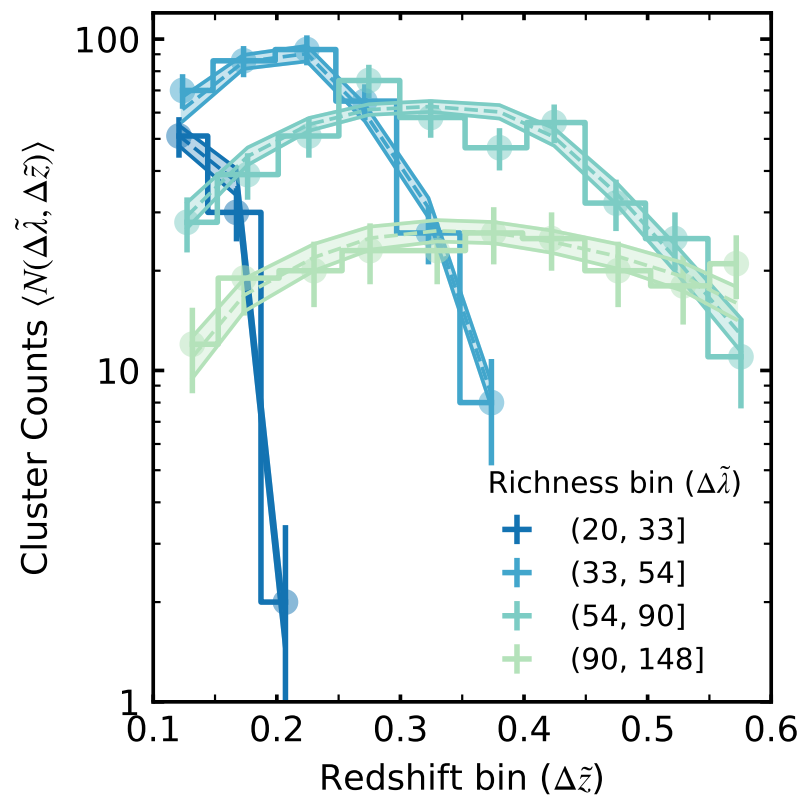

Figure B2. The abundance of simulated SPIDERS clusters as a function in bins of observed redshift $\left(\Delta \tilde{z}_{j}\right)$ and richness $\left(\Delta \ln \tilde{\lambda}_{i}\right)$ where $\tilde{z} \in[0.1,0.6)$ and $\tilde{\lambda} \in[25,148)$. Steps represent the simulated data (Fig. B1), the width and height correspond to the size of the bin and the magnitude of the diagonal (Poisson) elements of the covariance matrix, respectively. The position of the vertical bars indicates the mean redshift in each bin. The shaded regions trace the expectation value provided by the model (with a normal prior on the intrinsic scatter Bleem et al. 2020), centred on the median, which corresponds to the best-fit cosmology. The lower and upper limit are similarly set by the $15 \%$ and $85 \%$ confidence intervals. These distributions are calculated directly from the stored expectation values of cluster counts for the MCMC chains used to create the contours shown in Fig. B4

Table B1. Summary of measured parameters and their initial priors for the simulated SPIDERS DR16 dataset as shown in Fig. B4. The format of this table is identical to that of the observed SPIDERS DR16 data Table. 2. Fiducial values of the input Bleem et al. (2020) richness-mass relation are omitted as they are only defined for $\boldsymbol{M}_{500 \mathrm{c}}$ rather than $\boldsymbol{M}_{200 \mathrm{c}}$. For a comparison of the measured richness-mass relation relative to the input one, please refer to Fig. B3.

\begin{tabular}{cccc}
\hline \hline Parameter & Fiducial & Prior & Posterior \\
\hline$\Omega_{m_{0}}$ & 0.31 & $\in[0.1,0.8]$ & $0.32_{-0.06}^{+0.11}$ \\
$\sigma_{8}$ & 0.82 & $\in[0.4,1.2]$ & $0.84_{-0.06}^{+0.08}$ \\
$A$ & - & $\in[3.0,5.0]$ & $3.93_{-0.35}^{+0.19}$ \\
$B_{\mu}$ & - & $\in[0.0,10.0]$ & $1.10_{-0.21}^{+0.25}$ \\
$B_{z}$ & - & $\in[-5.0,5.0]$ & $0.08_{-0.81}^{+0.87}$ \\
$\sigma_{\text {int }}$ & 0.22 & $\sim \mathcal{N}(0.23,0.16)$ & $0.30_{-0.15}^{+0.11}$ \\
\hline
\end{tabular}

$\Omega_{m_{0}}$ represents the mean matter density of the universe at redshift zero.

$\sigma_{8}$ is the amplitude of the matter power spectrum.

$A$ is $\langle\ln \lambda(\mu, z)\rangle$ at pivot mass scale and pivot redshift.

$B_{\mu}$ is the coefficient of halo mass dependence in $\langle\ln \lambda(\mu, z)\rangle$.

$B_{z}$ is the coefficient of linear redshift dependence in $\langle\ln \lambda(\mu, z)\rangle$. $\sigma_{\text {int }}$ is the intrinsic scatter of the richness-mass relation. 

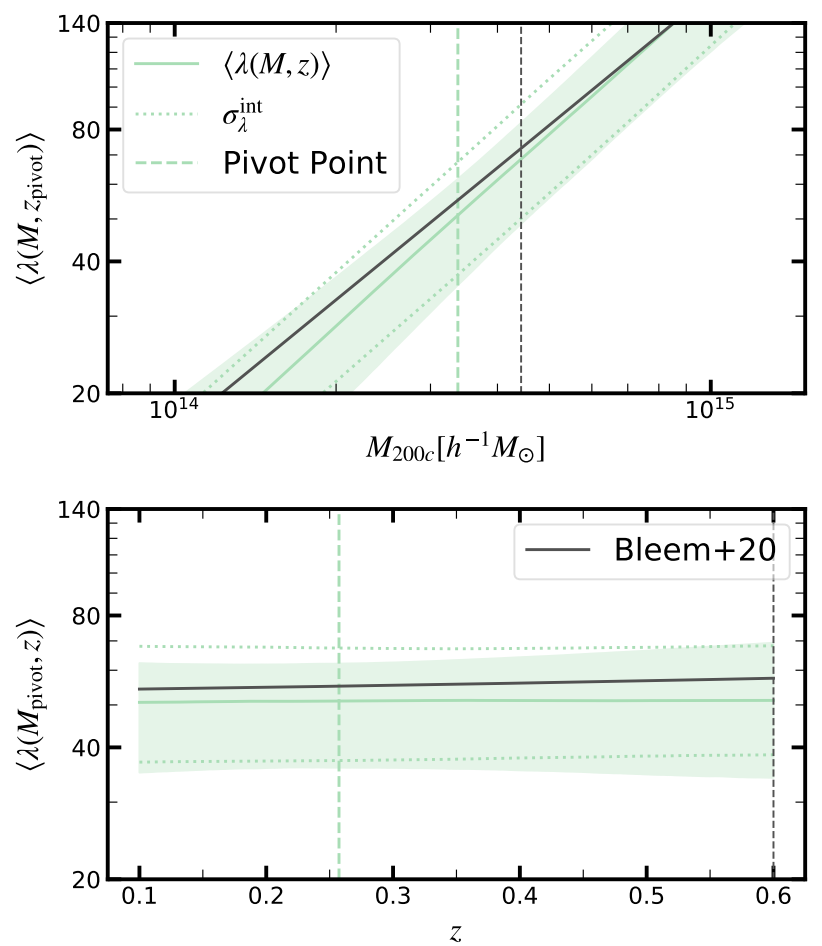

Figure B3. Constraints for the richness-mass relation evaluated at the pivot mass $M_{200 \mathrm{c}}=3.37 \times 10^{14} \mathrm{M}_{\odot} h^{-1}$ (lower panel) and pivot redshift 0.257 (upper panel) used in this analysis. The dotted line about the solid line indicates the intrinsic scatter (with normal prior from Bleem et al. 2020) about the mean (Eq. 2), while the coloured contours show the 16 and 85 percentiles of the draws from the MCMC chains used to constrain the cosmological and scaling relation parameters shown in Fig. B4 


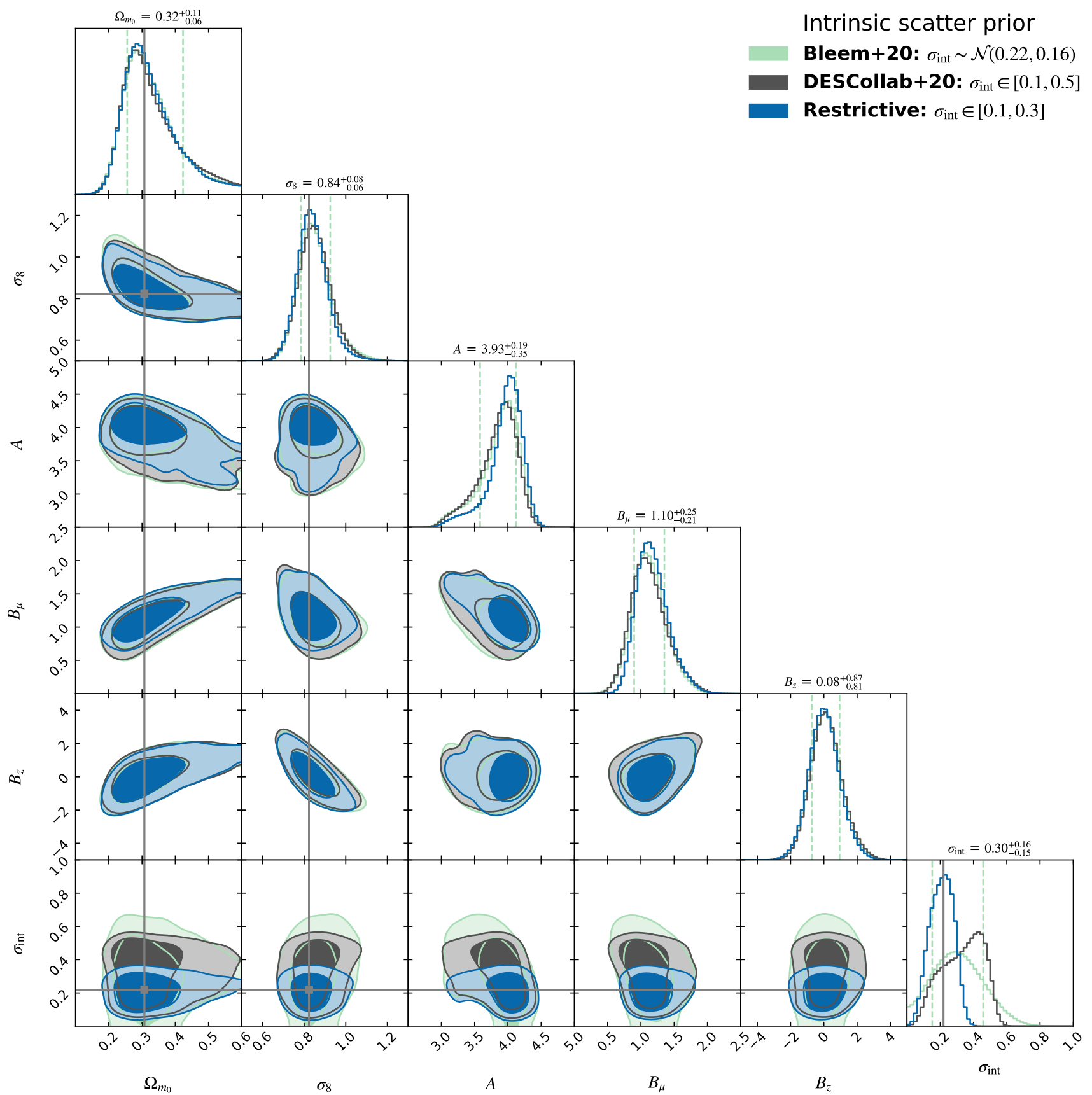

Figure B4. Cosmological parameters and scaling relation parameter constraints for the simulated SPIDERS DR16 dataset as summarised in Table. B1. Contours depict the one $68 \%$ and $95 \%$ confidence levels where posterior distributions are obtained using the full SPIDERS DR16 likelihood function outlined in Sect. 3.4. Input values of cosmological and intrinsic scatter parameters are marked by the grey lines, for a comparison of the input and recovered scaling relation, please refer to Fig. B3. The impact of the intrinsic scatter prior is shown by the different coloured posteriors (see legend). Although $\sigma_{\text {int }}$ is only well constrained when using the Bleem et al. (2020) prior, the determination of every other parameter is found to be relatively insensitive to the alternate priors. 